\title{
Anisotropy of seasonal snow measured by polarimetric phase differences in radar time series
}

\author{
Silvan Leinss ${ }^{1}$, Henning Löwe ${ }^{2}$, Martin Proksch ${ }^{2}$, Juha Lemmetyinen ${ }^{3}$, Andreas Wiesmann ${ }^{4}$, and Irena Hajnsek ${ }^{1,5}$ \\ ${ }^{1}$ Institute of Environmental Engineering, Swiss Federal Institute of Technology (ETH), Zurich, Switzerland \\ ${ }^{2}$ Institute for Snow and Avalanche Research SLF, Davos, Switzerland \\ ${ }^{3}$ Finnish Meteorological Institute FMI, Arctic Research, Sodankylä, Finland \\ ${ }^{4}$ GAMMA Remote Sensing AG, Gümlingen, Switzerland \\ ${ }^{5}$ Microwaves and Radar Institute, German Aerospace Center (DLR), Wessling, Germany
}

Correspondence to: Silvan Leinss (leinss@ifu.baug.ethz.ch)

Received: 1 October 2015 - Published in The Cryosphere Discuss.: 5 November 2015

Revised: 18 June 2016 - Accepted: 21 June 2016 - Published: 17 August 2016

\begin{abstract}
The snow microstructure, i.e., the spatial distribution of ice and pores, generally shows an anisotropy which is driven by gravity and temperature gradients and commonly determined from stereology or computer tomography. This structural anisotropy induces anisotropic mechanical, thermal, and dielectric properties. We present a method based on radio-wave birefringence to determine the depth-averaged, dielectric anisotropy of seasonal snow with radar instruments from space, air, or ground. For known snow depth and density, the birefringence allows determination of the dielectric anisotropy by measuring the copolar phase difference (CPD) between linearly polarized microwaves propagating obliquely through the snowpack. The dielectric and structural anisotropy are linked by MaxwellGarnett-type mixing formulas. The anisotropy evolution of a natural snowpack in Northern Finland was observed over four winters (2009-2013) with the ground-based radar instrument "SnowScat". The radar measurements indicate horizontal structures for fresh snow and vertical structures in old snow which is confirmed by computer tomographic in situ measurements. The temporal evolution of the CPD agreed in ground-based data compared to space-borne measurements from the satellite TerraSAR-X. The presented dataset provides a valuable basis for the development of new snow metamorphism models which include the anisotropy of the snow microstructure.
\end{abstract}

\section{Introduction}

After deposition on the ground, snow crystals form a porous, sintered material which continuously undergoes metamorphism to adapt to the thermodynamic forcing imposed by the atmosphere and the soil. The porous microstructure, defined by the 3-D distribution of the ice matrix and the pores space, determines the thermal, mechanical, and dielectric properties of the snowpack. Hence, a spatially anisotropic distribution of the snow microstructure leads to macroscopically anisotropic snow properties.

Characterization of the microstructure is difficult and requires work intensive sampling, sample preparation, and data processing but enables a unique insight into the structure at micrometer scales. Macroscopic (point) methods commonly applied in the field can be used to determine snow properties averaged over sample volumes of several centimeters. Methods based on remote sensing complement these point methods in providing large spatial coverage of repetitive measurements with a sampling resolution between meters and kilometers for inaccessible locations as well. For snow, radar remote sensing methods facilitate measurements of snow properties averaged over the microwave penetration depth. This makes it possible to estimate the depth-averaged dielectric anisotropy of seasonal snow with radar instruments. 


\subsection{Observations of the structural anisotropy}

Anisotropic structures have been identified in photographs of thin section cuts of seasonal snow with preferentially horizontal structures for fresh snow and vertical structures for old snow (Kojima, 1960; Davis and Dozier, 1989; Mätzler, 1987; Fig. $2.15^{1}$ ). Vertical structures have been reported also in thin section cuts of polar firn (e.g., Alley, 1987). The formation to anisotropic vertical snow structures has been observed by thin section photography, when snow metamorphism was driven by a vertical water vapor flux under temperature gradients (e.g., Pfeffer and Mrugala, 2002).

The anisotropy of snow can be statistically determined from the snow microstructure by spatial correlation functions (e.g., Vallese and Kong, 1981; Mätzler, 1997) computed from stereological (Alley, 1987; Mätzler, 2002, and others) or computer tomography (CT) data (e.g., Löwe et al., 2011, 2013). Today, CT is often considered as the "state of the art" for destruction-free observations of the microstructure within volumes of a few cubic centimeters and with a spatial resolution on the micrometer scale. The non-destructive CT measurements allow repeated imaging of a snow sample to observe the microstructural temporal evolution of samples kept under laboratory conditions (Schneebeli and Sokratov, 2004). The transformation to vertical structures from initially horizontal structures has been observed in laboratory samples when vertical temperature gradients have been applied (Schneebeli and Sokratov, 2004; Riche et al., 2013; Calonne et al., 2014). Vertical as well as horizontal structures have been found in artificial snow from the cold laboratory as well as in natural seasonal snow (Calonne et al., 2012). In polar firn, vertical structures are commonly found in firn cores at different depths (e.g., Hörhold et al., 2009; Fujita et al., 2009; Lomonaco et al., 2011).

In contrast to vertical structures, which are known to be caused by vertical temperature gradients, horizontal structures have a different origin. A horizontal orientation can be created by deposition of anisotropic, atmospheric growth forms (plates, needles, dendrites) which align predominantly horizontally in the gravity field (Matrosov et al., 2005; Garrett et al., 2012). Snow settling was also found to contribute to horizontal structures in the intermediate stage of isothermal metamorphism (Löwe et al., 2011).

\subsection{Field observations of the dielectric anisotropy}

On macroscopic scales, the anisotropy of snow can be characterized by measuring the anisotropy of the thermal conductivity (e.g., Izumi and Huzioka, 1975) or from the anisotropy of the dielectric permittivity (e.g., Fujita et al., 2016). The anisotropy of the thermal conductivity is much stronger compared to the anisotropy of the dielectric permittivity because the contrast in thermal conductivity $k$ between ice and air

\footnotetext{
${ }^{1}$ Note that the captions of Figs. 2.14 and 2.15 in Mätzler (1987) have been inadvertently swapped.
}

$k_{\text {ice }} / k_{\text {air }}$ is $\approx 100$ compared to the contrast in permittivity between ice and air, which is $\varepsilon_{\text {ice }} / \varepsilon_{\text {air }} \approx 3$ (Löwe et al., 2013). Still, dielectric measurements have been discussed already in 1965 with respect to the shape and orientation of ice crystals (Evans, 1965, and references therein).

The dielectric anisotropy, $\Delta \varepsilon$, defined in this paper as the difference between the horizontal and vertical permittivities, $\Delta \varepsilon=\varepsilon_{x}-\varepsilon_{z}$, can be measured precisely using different polarizations of the electromagnetic field. Using open microwave resonators of the design of Jones (1976), Matsuoka et al. $(1996,1997)$ measured the dielectric anisotropy of ice, which is caused by the $c$-axis orientation of the crystal fabric. With the same method, the dielectric anisotropy in snow, caused by a structural anisotropy of the ice matrix, has been measured and higher permittivities have been found in the vertical than the horizontal direction in multiyear firn on both the Greenland ice sheet (Fujita et al., 2014) and on the Antarctic ice sheet (Fujita et al., 2009, 2016); Fujita et al. (2009) did the analysis in conjunction with a CT analysis. Using a method of microwave propagation, Lytle and Jezek (1994) also detected a higher vertical than horizontal permittivity in multiyear firn on the Greenland ice sheet combined with a photographic analysis. Sugiyama et al. (2010) found similar results in Antarctica: the measured horizontal permittivity in the upper $1 \mathrm{~m}$ snow layer were often smaller than expected from empirical relations between permittivity and density of isotropic snow.

\subsection{Radio and microwave remote sensing observations of the dielectric anisotropy}

Polarimetric remote sensing methods can provide information about the dielectric anisotropy of snow and ice from large distances. Areas of many thousands of square kilometers can be observed with air- and space-borne sensors, even repeatedly by satellites during every repeat pass of an orbit. The imaging resolution and penetration depth of radio- or microwaves implies that usually area-, depth-, or volumeaveraged snow properties are measured. Remote sensing methods provide therefore a complementary tool to the detailed ground measurements such as CT or in situ measurements.

For example, radio-wave birefringence measurements have been used to explore internal structures of ice sheets and glaciers with polarized radio- and microwaves (e.g., Hargreaves, 1977, 1978; Fujita et al., 2006; Matsuoka et al., 2009; Parrella et al., 2016). With passive microwave sensors, strong polarimetric signatures have also been found over the Greenland ice sheet: in Li et al. (2008), the observed signatures could not be explained by surface features and were discussed with respect to microstructural variations of snow anisotropy, as predicted by Tsang (1991).

The observations of both structural and dielectric anisotropies in polar firn indicate that seasonal snow should also be a dielectrically anisotropic medium. Still, publi- 
cations related to polarimetric propagation effects in deposited seasonal snow are rare despite the fact that a differential propagation speed in falling snow was already noticed in 1976 for weather radars (Hendry et al., 1976). Today, polarimetric upward-looking radars are used to characterize the orientation and anisotropy of falling snow particles or rain (e.g., Matrosov et al., 2005; Garrett et al., 2012; Xie et al., 2012; Hogan et al., 2012; Noel and Chepfer, 2010; Tyynelä and Chandrasekar, 2014).

The dielectric anisotropy of seasonal snow can be determined from propagation delay differences of differently polarized microwaves transmitted obliquely through the snowpack. In contrast to observations on ice sheets where commonly $\varepsilon_{z}>\varepsilon_{x}$, a larger horizontal permittivity $\left(\varepsilon_{x}\right)$ was found in freshly deposited snow by ground-based radar measurements: Chang et al. (1996) measured the propagation delay between vertically and horizontally polarized microwaves by analyzing their phase difference, the so-called copolar phase difference (CPD). They found that the CPD increased after snowfall and explained this by a "horizontal alignment of new snow crystals" (Chang et al., 1996). In polarimetric measurements acquired by the radar satellite TerraSAR-X (TSX) (Stangl et al., 2006; Werninghaus and Buckreuss, 2010), both positive and negative CPDs were observed at different times by Leinss et al. (2014b): they show a positive correlation between the CPD and the depth of fresh snow, indicating horizontal structures with $\varepsilon_{x}>\varepsilon_{z}$. They also observed that strong temperature gradients decreased the CPD towards negative values, indicating the growth of vertical structures.

\subsection{Paper structure}

In this paper, we present and apply an electromagnetic model to determine the depth-averaged structural anisotropy of a dry snowpack from CPD measurements acquired by polarimetric radar systems. The paper is structured as follows.

- Sect. 2 revisits Maxwell-Garnett (MG) mixing formulas and also links them to the microstructural characterization of snow in terms of spatial correlation functions to calculate the anisotropic dielectric permittivity based on the structural anisotropy.

- Sect. 3 describes experimental and signal-processing considerations and derives the CPD expected to be measured for an anisotropic dielectric medium of known thickness and with negligible scattering effects.

- Sect. 4 describes the experiment and the field data collected within four winter seasons from 2009 to 2013. The CPD measurements are discussed in light of marker events like snowfall, snow metamorphism, and melting.

- Sect. 5 analyzes the CPD time series to determine the evolution of the depth-averaged anisotropy of the snowpack. For four selected dates, the derived anisotropy is compared with CT measurements. Furthermore, we compare the measured CPD time series with spaceborne radar observations from TSX and discuss whether the CPD can be used to determine the depth of fresh snow.

- Sect. 6 concludes the paper, followed by Appendix A-E to provide theoretical details and to list the author contributions for this collaborative paper.

\section{Dielectric permittivity linked to structural anisotropy}

The ice matrix of snow can have two different anisotropies, both of which can influence the anisotropy of the effective permittivity. The first, the structural anisotropy, is given by an anisotropic spatial distribution of the ice matrix, i.e., the shape of ice crystals. Below, we provide a relation between the structural anisotropy, snow density, and the effective permittivity, based on an adapted version of MG mixing formulas. The second, the crystal fabric anisotropy, is determined by the $c$-axis orientation of ice crystals but its effect on the permittivity of seasonal snow is small (Appendix A) compared to the effect of the structural anisotropy.

In the following we define the coordinate axes such that $z$ is the vertical (parallel to gravity) and $x-y$ is the horizontal plane. The model is restricted to flat terrain and does not consider the possibility that the symmetry axis of the microstructure is not vertical, as it could occur in steep terrain.

\subsection{Definition of structural anisotropy}

We define the structural anisotropy, $A$, as the normalized difference between the characteristic horizontal and vertical dimension, $a_{x}$ and $a_{z}$, of the "grains" in the ice matrix:

$A=\frac{a_{x}-a_{z}}{\frac{1}{2}\left(a_{x}+a_{z}\right)}$.

Different choices for the length scales $a_{x}$ and $a_{z}$ are possible (Löwe et al., 2011). Recent work for microwave modeling has mainly used the (exponential) correlation lengths, $a_{x}=p_{\mathrm{ex}, x}$ and $a_{z}=p_{\mathrm{ex}, z}$, as defined in Mätzler (2002). The exponential correlation lengths are conveniently derived by an exponential fit to spatial correlation functions (Löwe et al., 2013).

The advantage of the normalized difference, Eq. (1), is that $A$ only changes sign but not magnitude when the orientation of the longest length changes its orientation from vertical to horizontal (while keeping a fixed ratio between longest and shortest length). For the common definition $A^{\prime}$, where the anisotropy is defined by the length ratio $a_{z} / a_{x}$, the magnitude of the difference to the isotropic case $\left(A_{\text {iso }}^{\prime}=1\right)$ depends on the orientation of the longest length: the difference becomes clear when comparing, e.g., $\left(a_{x}=2, a_{z}=1\right)$, yielding $A=+0.66$ and $A^{\prime}=0.5=A_{\text {iso }}^{\prime}-0.5$, with $\left(a_{x}=1\right.$, $a_{z}=2$ ) yielding $A=-0.66$ and $A^{\prime}=2.0=A_{\text {iso }}^{\prime}+1.0$. The 
definition $A^{\prime}$ is commonly used to simplify electromagnetic modeling. The anisotropy $A^{\prime}$ can be converted to Eq. (1) by

$A^{\prime}=\frac{a_{z}}{a_{x}}=\frac{2-A}{2+A}$ or $A=\frac{1-A^{\prime}}{\frac{1}{2}\left[1+A^{\prime}\right]}$.

We note that the anisotropy $A^{\prime}$ differs from the definition in terms of the "degree of anisotropy" (DA) which is used in Hildebrand et al. (1999) and Schneebeli and Sokratov (2004). For the DA, the absolute orientation in space is lost since the definition is based on the ratio of the largest and smallest eigenvalues of the mean intersection length tensor. The anisotropy $A^{\prime}$ (defined as $\epsilon$ in Torquato and Lado, 1991, or $\mathcal{A}\left(l_{c}\right)$ in Calonne et al., 2014) can be further related to the anisotropy parameter $Q$ used in Calonne et al. (2014) by the definition in Löwe et al. (2013, Eq. 4).

\subsection{Relative permittivity as a function of anisotropic inclusions: Maxwell-Garnett formulas}

The CPD measured by polarimetric radar systems depends on the dielectric permittivities for the $x$ and $z$ direction. Therefore, this subsection establishes a link between the effective permittivities $\varepsilon_{\mathrm{eff}, i}$ for $i \in\{x, y, z\}$ and the structural anisotropy $A$. The following model is based on an empirical extension of the classical MG mixing formulas for aligned mixtures of elliptical inclusions in a host medium (e.g., Polder and van Santen, 1946; Sihvola, 2000).

To motivate the necessity of the empirical extension we briefly revisit the MG mixing formulas where the permittivity for isotropic ice inclusions in a host medium of air,

$\varepsilon_{\text {eff, } \mathrm{MG}}=\varepsilon_{\text {air }}+3 f_{\mathrm{vol}} \varepsilon_{\text {air }} \frac{\varepsilon_{\text {ice }}-\varepsilon_{\text {air }}}{\varepsilon_{\text {ice }}+2 \varepsilon_{\text {air }}-f_{\text {vol }}\left(\varepsilon_{\text {ice }}-\varepsilon_{\text {air }}\right)}$,

should agree with measurements of isotropic snow. However, $\varepsilon_{\text {eff, MG }}$ underestimates the permittivity measured in Mätzler (1996), which shows slightly higher values. The reason is that $\varepsilon_{\text {eff, MG }}$ is equivalent to the lower Hashin-Shtrikman bound (HSB) (Sihvola, 2002; Hashin and Shtrikman, 1962). The upper HSB is equivalent to the "inverse" MaxwellGarnett formula, $\varepsilon_{\text {eff, MG, inv }}$, which models air inclusions in a host medium of ice and follows from Eq. (3) by swapping the permittivities $\varepsilon_{\text {air }}=1$ and $\varepsilon_{\text {ice }} \approx 3.17$ and by replacing the ice volume fraction $f_{\mathrm{vol}}$ with $1-f_{\mathrm{vol}}$ (Sihvola, 2002). For better agreement with measured data, we combined both bounds in a reasonable way (see Appendix B) and obtained the following weighted average:

$\varepsilon_{\text {eff }}=\left(\varepsilon_{\text {eff,MG }}+\varepsilon_{\text {eff,MG,inv }} \cdot f_{\text {vol }} \varepsilon_{\text {ice }}\right) /\left(1+f_{\text {vol }} \varepsilon_{\text {ice }}\right)$.

The ice volume fraction $f_{\text {vol }}$ relates the density of snow $\rho$ $\left(\mathrm{g} \mathrm{cm}^{-3}\right)$ to the volumetric mass density of air and ice by

$\rho=f_{\mathrm{vol}} \cdot \rho_{\text {ice }}+\left(1-f_{\mathrm{vol}}\right) \cdot \rho_{\text {air }} \approx f_{\mathrm{vol}} \cdot \rho_{\text {ice }}$.

In the microwave regime between 10 and $20 \mathrm{GHz}$, the permittivity of pure polycrystalline ice shows only a weak tem- perature dependence: $\varepsilon_{\text {ice }}=3.17 \pm 0.02$ (Mätzler and Wegmüller, 1987; Fujita et al., 1993; Matsuoka et al., 1996; Warren and Brandt, 2008; Bohleber et al., 2012). As the uncertainty for snow density measurements of a few percent is larger than the temperature dependence of $\varepsilon_{\text {icee }}$, a fixed permittivity $\varepsilon_{\text {ice }}=3.17$ is used in this paper, corresponding to a temperature of about $-10^{\circ} \mathrm{C}$.

Note that the MG theory is a mean-field theory which additionally requires the inclusions to be much smaller than the wavelength $\lambda$ in the medium $\left(a_{x}, a_{y}, a_{z} \ll \lambda / \sqrt{\varepsilon_{\text {eff }}}\right)$ to neglect scattering in the snow volume.

For non-spherical inclusions, Eq. (3) has to be adapted by introducing depolarization factors, $N_{\mathrm{i}}$, for aligned ellipsoidal inclusions (e.g., Cohn, 1900; Polder and van Santen, 1946, or Sihvola, 2000). As settling and temperature gradients act in the $z$ direction, we model the inclusions as oblate or prolate spheroids which have their symmetry axis parallel to $z$. According to Sihvola (2000) the permittivity of anisotropic mixtures is given for each spatial dimension $i \in x, y, z$ by

$\varepsilon_{\mathrm{eff}, \mathrm{MG}, i}=\varepsilon_{\mathrm{air}}+f_{\mathrm{vol}} \varepsilon_{\mathrm{air}} \frac{\varepsilon_{\text {ice }}-\varepsilon_{\text {air }}}{\varepsilon_{\text {air }}+\left(1-f_{\mathrm{vol}}\right) N_{\mathrm{i}}\left(\varepsilon_{\text {ice }}-\varepsilon_{\mathrm{air}}\right)}$.

The "inverse" MG form of Eq. (6a) reads

$\varepsilon_{\text {eff,MG,inv }, i}=\varepsilon_{\text {ice }}+\left(1-f_{\text {vol }}\right) \varepsilon_{\text {ice }} \frac{\varepsilon_{\text {air }}-\varepsilon_{\text {ice }}}{\varepsilon_{\text {ice }}+f_{\text {vol }} N_{\mathrm{i}}\left(\varepsilon_{\text {air }}-\varepsilon_{\text {ice }}\right)}$.

Both equations are used in Eq. (4) to calculate the effective anisotropic permittivities, $\varepsilon_{\mathrm{eff}, x}$ and $\varepsilon_{\mathrm{eff}, z}$. Figure 1 shows the obtained permittivities for isotropic and anisotropic cases.

The depolarization factors $N_{\mathrm{i}}$ are assumed to be equivalent in Eqs. (6a) and (6b) as both of them describe the polarizability of elliptical inclusion. The factors $N_{\mathrm{i}}$ are given according to Sihvola (2000) for ellipsoidal inclusions with the dimensions $a_{x}, a_{y}, a_{z}$ by the elliptic integral of the second kind:

$$
N_{\mathrm{i}}=\frac{a_{x} a_{y} a_{z}}{2} \int_{0}^{\infty} \frac{\mathrm{d} s}{\left(s+a_{i}^{2}\right) \sqrt{\left(s+a_{x}^{2}\right)\left(s+a_{y}^{2}\right)\left(s+a_{z}^{2}\right)}},
$$

where the integration variable $s$ (units: distance square) describes an ellipsoidal surface larger than the surface of the elliptic inclusion on which $s=0$ (Landau and Lifshitz, 1960, Sect. 4, 20-30). The dimensions $a_{x}=a_{y}$ define the (horizontal) diameter of the spheroids and $a_{z}$ is their vertical length. Note that Sihvola (2000) used the ellipsoids' semiaxis. However, the depolarization factors do not depend on the absolute size of inclusions and are invariant under rescaling $a_{i} \rightarrow \lambda a_{i}$ for arbitrary $\lambda$. Consequently, it is possible to parameterize the depolarization factors directly by the anisotropy $A^{\prime}$, which can easily be verified by substituting $s$ in Eq. (7) with the dimensionless quantity $u=s / a_{x}^{2}$ :

$$
N_{\mathrm{i}}=\frac{A^{\prime}}{2} \int_{0}^{\infty} \frac{\mathrm{d} u}{\left(u+\delta_{A^{\prime}}(i, z)\right) \sqrt{(u+1)^{2} \cdot\left(u+A^{\prime 2}\right)}},
$$



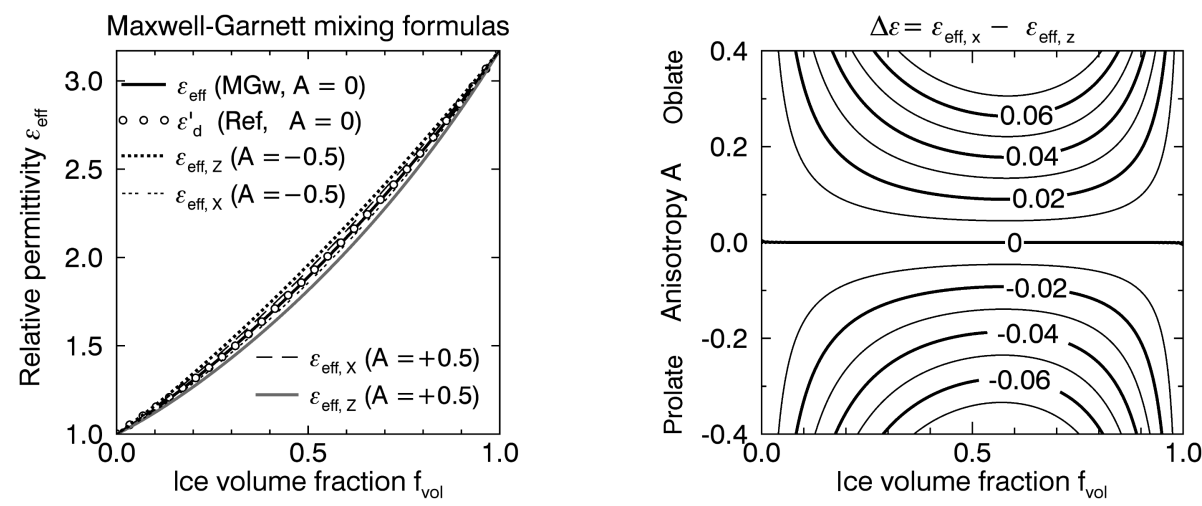

Figure 1. Left panel: relative permittivity $\varepsilon_{\text {eff }}$ of dry snow with isotropic $(A=0$, solid), vertically $(A=-0.5$, dots), and horizontally oriented ( $A=+0.5$, dashed) inclusions calculated by the weighted Maxwell-Garnett formula (MGw), Eq. (4). Open circles indicate the empirical

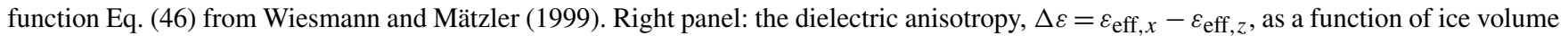
fraction $f_{\mathrm{vol}}$ and anisotropy $A$ according to Eq. (4).

with $\delta_{A^{\prime}}(i, z)=1$ for $i \in x, y$ and $\delta_{A^{\prime}}(i, z)=A^{\prime 2}$ for $i=z$. Closed form expressions for the elliptic integrals can be found, e.g., in Landau and Lifshitz (1960) and Sihvola (2000). The depolarization factors satisfy $N_{x}+N_{y}+N_{z}=1$ for any ellipsoid (Polder and van Santen, 1946). For spherical inclusions all three depolarization factors are $N_{\mathrm{i}}=1 / 3$ and Eq. (6a) is equivalent to Eq. (3).

\subsection{Series expansion of permittivity via correlation functions: equivalence to Maxwell-Garnett}

Although ice grains show a much more complex structure than simple ellipsoids, the model of ellipsoids is realistic enough for the transverse isotropic symmetry of the dielectric tensor $\overline{\bar{\varepsilon}}$. This becomes more obvious from the exact series expansion of the dielectric tensor for arbitrary anisotropic microstructures, which can be expressed in terms of spatial correlation functions (Rechtsman and Torquato, 2008). In Appendix C, we show that under the less restrictive assumption of a transverse isotropic two-point correlation function, the truncation of the exact expression using $n$ point correlation functions (Rechtsman and Torquato, 2008, Eq. 16) at second order $(n=2)$ exactly leads to the MG result (Eq. 6a) in which the depolarization factors $N_{\mathrm{i}}$ are expressed in terms of the anisotropy parameter $Q$ as given in Löwe et al. (2013) via $N_{\mathrm{i}}=Q$ for $i=x, y$ and $N_{z}=1-2 Q$. This implies that the present dielectric model and the thermal conductivity model from Löwe et al. (2013) are based on exactly the same microstructural parameters. In light of recent attempts to unify microstructural descriptions of snow for microwave modeling (Löwe and Picard, 2015), we also note that the MG formula (Eq. 6a) can be likewise obtained as the lowfrequency limit of the quasi-crystalline approximation for aligned spheroids (Ao and Kong, 2002).

\section{Dielectric anisotropy measured by polarimetric radar systems}

The depth-averaged anisotropy of a snowpack of known depth and density can be measured with an obliquely looking polarimetric radar system when the phase difference between two perpendicular polarized microwaves pulses reflected at the bottom of the snowpack is analyzed with respect to the microwave birefringence of snow.

\subsection{Experimental considerations}

For measuring quantitatively the dielectric anisotropy of the snowpack, the angle between the electromagnetic field vector and the principal axes of the dielectric tensor $\overline{\bar{\varepsilon}}$ must be known. Therefore, the polarizations of two orthogonally polarized microwaves should be chosen such that the polarizations are delayed by different components of the dielectric tensor $\overline{\bar{\varepsilon}}$. The anisotropy of seasonal snow has its symmetry axis in the vertical; therefore one polarization must be at least partially aligned with the vertical while the other polarization must be oriented horizontally. Side-looking polarimetric radar systems, such as real or synthetic aperture radar systems using a vertical (VV) and horizontal ( $\mathrm{HH})$ polarization as defined in Fig. 2, fulfill this requirement, whereas the anisotropy cannot be measured by nadir-looking radar systems (e.g., ground-penetrating radars) as long as there is no anisotropy in the horizontal $(x y)$ plane.

Furthermore, the depth must be known where most of the microwave energy is scattered back. For dry snow and frequencies of a few $\mathrm{GHz}$ where the volume scattering contribution in shallow seasonal snow is negligible (e.g., Hallikainen et al., 1987; West et al., 1993; Tsang et al., 2007, or Leinss et al., 2015, Fig. 5), this requirement is easy to fulfill and the scattering center corresponds to the soil below the snowpack. However, for deep firn on ice sheets or glaciers it can be difficult to obtain a good estimate on the penetration depth. 


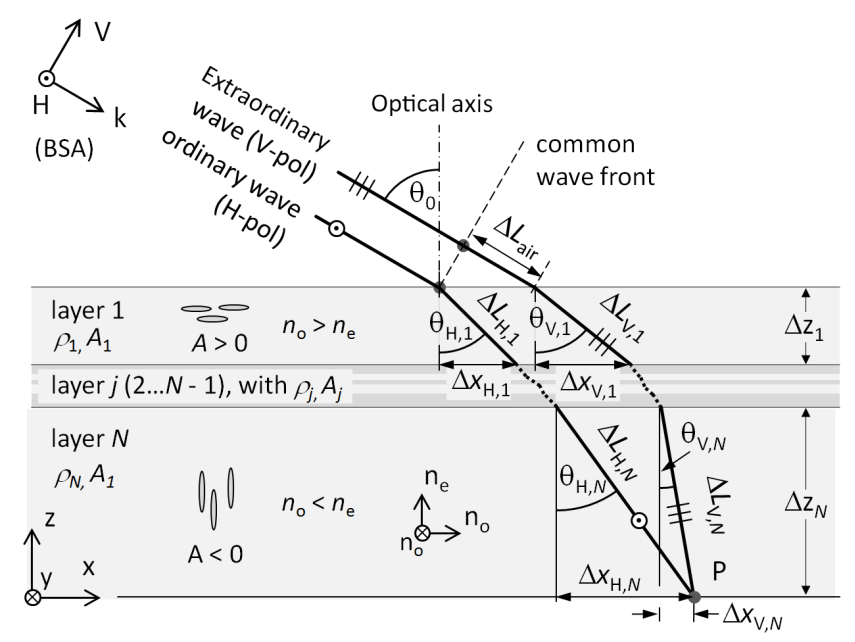

Figure 2. An electromagnetic wave (H- or V-polarized) is transmitted in $\boldsymbol{k}$ direction with respect to the radar coordinate system $(H, k, V)$ and with an incidence angle $\theta_{0}$ with respect to the snow surface. The electric field of the H-polarized wave is perpendicular to the optical axis $(z)$ and sees the ordinary refractive index $n_{\mathrm{O}}$ (therefore called the "ordinary wave"). The electric field of the Vpolarized wave has a component parallel to the optical axis and is affected by the extraordinary refractive index $n_{\mathrm{e}}$ (the "extraordinary wave"). For horizontally aligned structures $(A>0)$ the extraordinary wave travels faster $\left(n_{\mathrm{e}}<n_{\mathrm{O}}\right)$ whereas for vertical structures the ordinary wave is faster $\left(n_{\mathrm{o}}<n_{\mathrm{e}}\right)$. As refraction differs for both waves, the optical distances also differ when measured from a common wave front to the same point $P$ on the ground. Describing the radar reflection at a common point $P$ seems arbitrarily chosen, but only reflections from common scatterers within one radar resolution cell contribute to the CPD as described in Sect. 3.2. The structural anisotropy of the layers is shown as expected for fresh snow (layer 1) deposited on top of old snow (layer $N$ ). The layer of fresh snow with density $\rho_{1}$ and thickness $\Delta z_{1}$ is drawn with horizontal structures with a anisotropy $A>0$. The thick layer of old snow is drawn as vertical ice grains $(A<0)$ grown by temperature gradient metamorphism. The theory in this paper is true for any random layering of densities and anisotropies due to Snell's law as long as absorption and volume scattering are negligible.

The following method is not suitable for wet snow, as the dielectric properties, especially absorption and the penetration depth, strongly depend on the water content.

\subsection{CPD definition and copolar coherence}

The CPD can be used to measure the propagation delay difference of two orthogonally polarized microwaves. The CPD is defined as the phase difference,

$\phi_{\mathrm{CPD}}=\phi_{\mathrm{VV}}-\phi_{\mathrm{HH}}$,

between the phase values $\phi_{\mathrm{VV}}$ and $\phi_{\mathrm{HH}}$ of the complex radar backscatter coefficients, $S_{\mathrm{VV}}$ and $S_{\mathrm{HH}}$. The scattering coefficient for the vertical polarization, $S_{\mathrm{VV}}$ (VV being V transmit, $\mathrm{V}$ receive), is determined by the coherent superposi- tion of all scattered fields from the ensemble of scatterers contained in the corresponding range-resolution cell of the radar (the pixel). The random distribution of scatterers in the resolution cell defines the unknown but deterministic scattering-phase $\phi_{\mathrm{VV}}$. The scattering coefficient $S_{\mathrm{HH}}$ is equivalently defined for perpendicular transmit $(\mathrm{H})$ and receive polarization $(\mathrm{H})$. If the scatterers do not have a polarization dependent scattering phase and if no birefringent medium exists between the radar and the scatterers, the (generally random) phase values $\phi_{\mathrm{VV}}$ and $\phi_{\mathrm{HH}}$ are spatially correlated and show a zero-phase difference. For scatterers with homogeneously distributed amplitudes, the CPD is equivalent with the phase $\phi_{\mathrm{CPD}}^{\gamma}$ of the copolar coherence, given by the (complex-valued) spatial correlation function

$\gamma_{\mathrm{VV}, \mathrm{HH}} \cdot e^{i \phi_{\mathrm{CPD}}^{\gamma}}=\frac{\left\langle S_{\mathrm{VV}} \cdot S_{\mathrm{HH}}^{*}\right\rangle}{\sqrt{\left\langle\left|S_{\mathrm{VV}}\right|^{2}\right\rangle \cdot\left\langle\left|S_{\mathrm{HH}}\right|^{2}\right\rangle}}$.

The magnitude of the copolar coherence defines the correlation coefficient $\gamma \mathrm{VV}$,HH ranging from 0 to 1 . The notation $\langle\cdot\rangle$ indicates a spatial average over about 10 to several thousands of pixels containing the backscatter coefficients of each polarization, and the asterisk * denotes complex conjugation.

The magnitude of the coherence is reduced when two corresponding resolution cells (of same range but different polarization) contain scatterers which do not show a correlation between orthogonal polarizations. This is the case for objects showing strong multiple scattering (e.g., rough surfaces and strongly scattering volumes). The coherence is also reduced when the corresponding range-resolution cells, represented by $S_{\mathrm{VV}}$ and $S_{\mathrm{HH}}$, are not perfectly overlapping and therefore do not contain exactly the same ensemble of scatterers. This occurs, for example, for large propagation delays $\Delta R$ between the two polarizations. For partially overlapping resolution cells of size $\delta r$, the coherence is reduced proportional to $1-\Delta R / \delta r$. The coherence is totally lost when $\Delta R$ exceeds the range resolution $\delta r$ of the radar system. For partially overlapping resolution cells, only scatterers which are contained in the overlapping parts of the resolution cells of different polarizations contribute constructively to the coherence; other scatterers lead to decorrelation. The contribution of correlated scatterers to the CPD can therefore be described by two polarized waves which have a common wave front before propagating through a birefringent medium and which are scattered at exactly the same point $P$ on the ground. This scattering geometry is the basis of Fig. 2 .

\subsection{Sign of CPD in backscatter alignment convention}

For monostatic radar systems, the transmitted and received waves are described in same coordinate system $(\mathrm{H}, k, \mathrm{~V}$; see Fig. 2), which is called the "backscatter alignment" (BSA) convention (Lüneburg and Boerner, 2004, Lee and Pottier, 2009, Sect. 3.1.3, or Cloude, 2010, Sect. 1.4.2). The BSA results in a reversal of the wave vector $\boldsymbol{k}$ in the receiving coordinate system with respect to the propagation direction. 
The reversal of $\boldsymbol{k}$ causes a sign change of the CPD; hence the physically expected phase difference $\phi_{\mathrm{CPD}}^{\prime}$ caused by birefringent media is related to the phase difference measured in the BSA by

$\phi_{\mathrm{CPD}}=(-1) \cdot \phi_{\mathrm{CPD}}^{\prime}$.

\subsection{CPD of birefringent, non-scattering media}

The CPD, being a phase of a signal, allows precise measurement of a dielectric anisotropy because it can be determined with a precision of a few degrees (i.e., fraction of one wavelength) relative to the total phase delay of many wavelengths, which is accumulated during propagation through the snowpack (Guneriussen et al., 2001, Eq. 5, and Leinss et al., 2015, Eq. 14). For example, for $1 \mathrm{~m}$ snow of density $\rho=0.25 \mathrm{~g} \mathrm{~cm}^{-3}$ a dielectric anisotropy $\Delta \varepsilon=\varepsilon_{x}-\varepsilon_{z}=10^{-4}$ causes a CPD of $1^{\circ}$ relative to the total phase delay of $5700^{\circ}$ measured at a radar frequency of $10 \mathrm{GHz}$ at $40^{\circ}$ incidence angle. The dielectric anisotropy of transparent media (e.g., a dry snowpack) can therefore be measured much more accurately with the CPD compared to the time delay between two perpendicularly polarized microwave pulses.

In order to derive the $\mathrm{CPD}$, the wave propagation through snow is formulated in analogy to transversely isotropic media as done in anisotropic optics (Saleh and Teich, 1991). Considering snow as transversely isotropic is reasonable since gravity and the direction of the water vapor flux in snow break isotropy in the vertical direction; therefore the optical axis is given by the $z$ axis.

According to anisotropic optics, we define the refractive index in the $z$ direction as the extraordinary refractive index, $n_{\mathrm{e}}$. For transversely isotropic media, the extraordinary refractive index differs from the ordinary refractive indices $n_{\mathrm{o}}$, which is defined in the $(x, y)$ plane. The refractive indices are related to the permittivity, Eqs. (4), (6a), and (6b), by

$n_{\mathrm{o}}^{2}=\varepsilon_{\mathrm{eff}, x}=\varepsilon_{\mathrm{eff}, y}$,

$n_{\mathrm{e}}^{2}=\varepsilon_{\mathrm{eff}, z}$.

The polarizations of side-looking radar systems are defined orthogonal to the propagation vector $\boldsymbol{k}$ of the incident beam such that the $\mathrm{H}$ polarization is oriented parallel to the observed surface (Fig. 2). Hence, the propagation velocity of the $\mathrm{H}$ polarization is determined by the ordinary refractive index $n_{0}$.

The $\mathrm{V}$ polarization is defined perpendicular to $\mathrm{H}$ and $\boldsymbol{k}$. Note that the V-polarized field is never parallel to the optical axis $z$ because the incidence angle $\theta_{0}$ is smaller than $90^{\circ}$ for side-looking radar systems. Therefore, the V-polarized field always has one component parallel to the optical axis $z$ and one component perpendicular to it, along $x$. For the $\mathrm{V}$ polarization, the refractive index $n_{\mathrm{V}}$ depends on the propagation angle $\theta_{\mathrm{V}}$ in the medium and can be described by the refractive index ellipsoid (Saleh and Teich, 1991):
$\frac{1}{n_{\mathrm{V}}^{2}\left(\theta_{\mathrm{V}}\right)}=\frac{\cos ^{2} \theta_{\mathrm{V}}}{n_{\mathrm{o}}^{2}}+\frac{\sin ^{2} \theta \mathrm{v}}{n_{\mathrm{e}}^{2}}$

The refractive indices for the $\mathrm{H}$ - and $\mathrm{V}$-polarized waves are ${ }^{2}$

$n_{\mathrm{H}}=n_{\mathrm{o}}$,

$n_{\mathrm{V}}\left(\theta_{\mathrm{V}}\right)=\frac{n_{\mathrm{o}} n_{\mathrm{e}}}{\sqrt{n_{\mathrm{e}}^{2} \cos ^{2} \theta_{\mathrm{V}}+n_{\mathrm{o}}^{2} \sin ^{2} \theta}}$.

The refraction at the air-snow interface is described by Snell's law which for the $\mathrm{H}$ polarization is

$n_{\text {air }} \sin \theta_{0}=n_{\mathrm{H}} \sin \theta_{\mathrm{H}}$

For the $\mathrm{V}$ polarization, the refractive index $n_{\mathrm{V}}$ depends on $\theta_{\mathrm{V}}$, which in turn depends on $n_{\mathrm{V}}$. The modified Snell's law

$n_{\text {air }} \sin \theta_{0}=n_{\mathrm{V}}\left(\theta_{\mathrm{V}}\right) \sin \theta_{\mathrm{V}}$

must therefore be solved simultaneously with Eq. (14b). It follows that

$n_{\mathrm{V}}\left(\theta_{\mathrm{V}}\right)=\sqrt{n_{\mathrm{o}}^{2}+\left(1-\frac{n_{\mathrm{o}}^{2}}{n_{\mathrm{e}}^{2}}\right) n_{\mathrm{air}}^{2} \sin ^{2} \theta_{0}}$.

Equation (16) can be used in Eq. (15b) to calculate the angle $\theta_{\mathrm{V}}$. Note that $\theta_{\mathrm{V}}$ is only implicitly contained in Eq. (16) by $\theta_{0}$ and Snell's law (Eq. 15b). For a birefringent medium, $\theta_{\mathrm{V}}$ no longer describes the direction of propagation of an optical beam (which does the Poynting vector) but instead the direction, which is perpendicular to the wave fronts (the wave vector $\boldsymbol{k}$ ). As we are interested in the retardation of wave fronts, we use $\theta_{\mathrm{V}}$ which determines the direction of $\boldsymbol{k}$ in the birefringent medium. The propagation angle $\theta_{j}$ within each layer $j$ of multilayer systems comprising $N$ anisotropic layers, all of which have the optical axis parallel to $z$, follows directly from Eqs. (15a) and (15b) because Snell's law holds at each layer interface, i.e.,

$n_{j} \sin \theta_{j}=n_{j+1} \sin \theta_{j+1}$ for $j=0,1 \ldots N-1$,

where $n_{0}=n_{\text {air }} \approx 1$.

The CPD for a multilayer system with arbitrary refractive indices can now be calculated. Figure 2 shows the geometry where each layer of thickness $\Delta z_{j}$ can have a different anisotropy $A_{j}$ and density $\rho_{j}$. Two perpendicular polarized plane waves of frequency $\nu=\omega /(2 \pi)$ described by $\boldsymbol{E}(t$, $\boldsymbol{r})=\boldsymbol{E}_{0} e^{i(\omega t-\boldsymbol{k})}$ are transmitted to the snow surface with an incidence angle $\theta_{0}$. For a fixed time $t$, the accumulated phase

\footnotetext{
${ }^{2}$ Note that the equations for $n_{\mathrm{V}}^{2}$ in Leinss et al. (2014b) and Cloude (2010, Eq. 3.135)) are approximations of Eq. (13) for small anisotropies. The approximations follow from Eq. (13) by writing $n_{\mathrm{o}}^{2}=\epsilon_{\text {eff }}-\delta$ and $N_{\mathrm{i}}^{2}=\epsilon_{\text {eff }}+\delta$ and applying a first-order Taylor expansion in $\delta$, neglecting terms $\mathcal{O}\left(\delta^{2} / \epsilon_{\text {eff }}^{2}\right)$.
} 

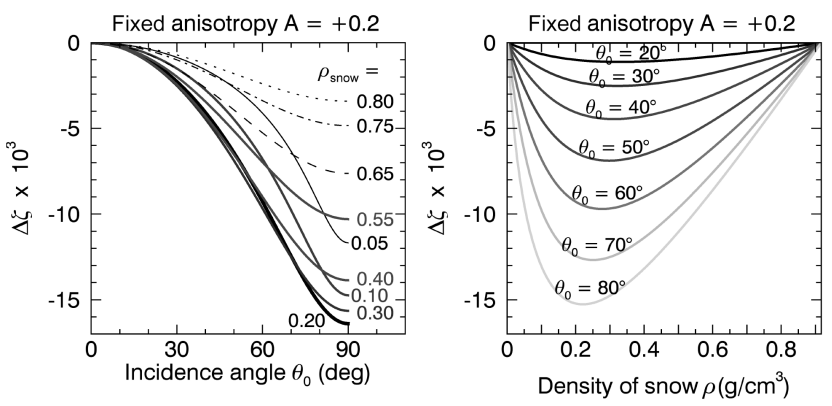

Figure 3. Specific path length difference between $\mathrm{V}$ - and $\mathrm{H}-$ polarized microwaves $(\Delta \zeta$, Eq. 23$)$ plotted for snow with horizontally aligned oblate ice grains $(A=+0.2)$ over incidence angle (left panel) and snow density (right panel).

along a distance $r$ is given by $\phi=\boldsymbol{k} \cdot \boldsymbol{r}$, where the magnitude $k=|\boldsymbol{k}|$ of the ordinary $(\mathrm{H})$ and extraordinary $(\mathrm{V})$ wave vectors depends on the vacuum wavelength $\lambda_{0}$ and the corresponding refractive indices:

$k_{\mathrm{H}}=\frac{2 \pi n_{\mathrm{H}}}{\lambda_{0}}$ and $k_{\mathrm{V}}=\frac{2 \pi n_{\mathrm{V}}}{\lambda_{0}}$.

The two paths for the ordinary and extraordinary waves, which connect a common wave front with a point $P$ at the snow-soil interface, are drawn in Fig. 2. The two-way phase difference between the two paths is given by

$$
\begin{aligned}
\phi_{\mathrm{CPD}}^{\prime} & =2 \cdot \sum_{j=1}^{N}\left(k_{\mathrm{V}, j} \Delta L_{\mathrm{V}, j}-k_{\mathrm{H}, j} \Delta L_{\mathrm{H}, j}\right)+2 k_{0} \Delta L_{\mathrm{air}} \\
& =2 \sum_{j=1}^{N} \frac{k_{\mathrm{V}, j} \Delta z_{j}}{\cos \theta_{\mathrm{V}, j}}-2 \sum_{j=1}^{N} \frac{k_{\mathrm{H}, j} \Delta z_{j}}{\cos \theta_{\mathrm{H}, j}}+2 \phi_{\mathrm{air}} .
\end{aligned}
$$

The in-air phase difference $\phi_{\text {air }}=k_{0} \Delta L_{\text {air }}$ depends on the sum of horizontal displacements $\sum \Delta x_{\mathrm{H}, j}-\Delta x_{\mathrm{V}, j}$ and the wave vector in air, $k_{0}=2 \pi n_{\text {air }} / \lambda_{0}$, and is given by

$\phi_{\text {air }}=-k_{0} \cdot \sin \theta_{0} \sum_{j=1}^{N} \Delta z_{j}\left(\tan \theta_{\mathrm{v}, j}-\tan \theta_{\mathrm{H}, j}\right)$.

Equation (20) can be rearranged and combined with Eqs. (21) and (18) to formulate the CPD in the BSA convention Eq. (11) as

$\phi_{\mathrm{CPD}}=(-1) \frac{4 \pi}{\lambda_{0}} \sum_{j=1}^{N} \Delta z_{j} \cdot \Delta \zeta\left(\rho_{j}, A_{j}, \theta_{0}\right)$.

In Eq. (22), the contribution each layer of thickness $\Delta z$ to the total CPD is determined by the specific path length difference

$\Delta \zeta\left(\rho, A, \theta_{0}\right)=\sqrt{n_{\mathrm{V}}^{2}-\sin ^{2} \theta_{0}}-\sqrt{n_{\mathrm{H}}^{2}-\sin ^{2} \theta_{0}}$,

which defines the optical path length difference per $\Delta z$ for an anisotropic medium, observed under the surface incidence angle $\theta_{0}$. The refractive indices $n_{\mathrm{V}}$ and $n_{\mathrm{H}}$, Eqs. (14a)
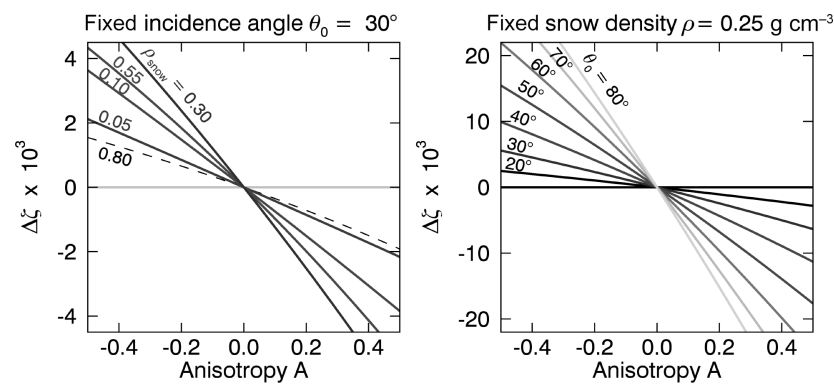

Figure 4. Specific path length difference $\Delta \zeta$ plotted over anisotropy for different snow densities but a fixed incidence angle, $\theta_{0}=30^{\circ}$ (left panel), and for different incidence angles but a fixed snow density, $\rho=0.25 \mathrm{~g} \mathrm{~cm}^{-3}$ (right panel).

and (16), are defined by the permittivities Eqs. (12a) and (12b) derived in Sect. 2.2 for given snow density $\rho$ and anisotropy $A$. For example, fresh snow with horizontal structures $\left(A>0\right.$; thus $\varepsilon_{x}>\varepsilon_{z}$ (Fig. 1) and $\left.n_{\mathrm{H}}>n_{\mathrm{V}}\right)$ causes a slower propagation for the $\mathrm{HH}$ polarization than for VV. Hence, HH will have a larger phase delay than $\mathrm{VV}$ at the receiving antenna, resulting in a positive CPD, $\phi_{\mathrm{CPD}}=\phi_{\mathrm{VV}}-\phi_{\mathrm{HH}}$, due to the sign change from the BSA.

The specific path length difference $\Delta \zeta$ increases with incidence angle and snow density until $\rho \approx 0.2 \mathrm{~g} \mathrm{~cm}^{-3}$ (Fig. 3). Above $\rho \approx 0.3 \mathrm{~g} \mathrm{~cm}^{-3}$, refraction reduces the alignment of the V-polarized field with respect to the optical axis such that $\Delta \zeta$ decreases. The resulting broad minimum (Fig. 3, right panel) causes a weak density dependence for the density range of seasonal snow. For seasonal snow, densities between 0.15 and $0.4 \mathrm{~g} \mathrm{~cm}^{-3}$ have been reported (Bormann et al., 2013). Within this range, the CPD varies by less than $20 \%$. This weak density dependence allows for quite rough density estimation when the CPD is used to determine the anisotropy of seasonal snow. In contrast to the weak density dependence, $\Delta \zeta$ depends for all densities and incidence angles almost linearly on anisotropy $A$ (Fig. 4). Thus, the CPD is mainly determined by snow depth $\Delta z$ and anisotropy $A$, which makes determination of $A$ almost independent on snow density.

Above $\rho>0.55$, the dielectric anisotropy $\Delta \varepsilon=\varepsilon_{x}-\varepsilon_{z}$ decreases (Fig. 1) such that $\Delta \zeta$ vanishes at $\rho=\rho_{\text {ice }}$ where no air inclusions are present anymore. We note that $\Delta \zeta$ vanishes only for isotropic (polycrystalline) ice. This is not generally the case as for ice on glaciers and ice sheets the crystal axis of ice ( $c$ axis) can have a preferential orientation (e.g., Matsuoka et al., 1997; Fujita et al., 2014, and Appendix A).

\subsection{Discussion of the CPD regarding literature results}

For firn (with $\rho=0.4 \mathrm{~g} \mathrm{~cm}^{-3}, \Delta \varepsilon=-0.05$ ), as observed by Fujita et al. (2014) for the upper $5 \mathrm{~m}$ of the ice sheet at NEEM (Northwest Greenland), we would expect a negative anisotropy $A=-0.25\left(A^{\prime}=1.3\right)$ due to vertical struc- 
tures. Similar firn conditions $\left(\rho=0.4 \mathrm{~g} \mathrm{~cm}^{-3}, \Delta \varepsilon=-0.05\right)$ have been found by Fujita et al. (2009) at Dome Fuji in Antarctica, who determined a slightly lower structural anisotropy $\left(A^{\prime} \approx 1.15\right)$ by means of X-ray microtomography. Similar values, $A^{\prime}=1.2$ and larger, have been observed in Antarctic firn by Alley (1987). In a recent firn core study from Dome Fuji, measurements of dielectric anisotropies $(\Delta \varepsilon=-0.01 \ldots-0.05)$ were used as a surrogate for a geometrically anisotropic microstructure (Fujita et al., 2016). The density and dielectric measurements of the studies of Fujita indicate that a CPD of $\phi_{\mathrm{CPD}}=-80^{\circ}$ per meter would have been measured for the radar parameters of the satellite TSX as used in the following study on seasonal snow: in Leinss et al. (2014b) a CPD of $60-150^{\circ} \mathrm{m}^{-1}$ was measured for fresh snow $(\rho=0.2)$ in Finland at $\theta_{0}=32.7^{\circ}$ and $9.65 \mathrm{GHz}$, corresponding to elongated horizontal structures with an anisotropy between $A=+0.2$ and $+0.5\left(A^{\prime-1}=1.2\right.$ and 1.7). Somewhat lower anisotropy values were found for natural, undisturbed, and also for sieved seasonal snow where in both cases initially horizontal features $\left(A^{\prime-1}=1.12\right.$ and 1.17) decayed within 6 days, after which in one case a preferentially vertical orientation $\left(A^{\prime}=1.12\right)$ was found (Schneebeli and Sokratov, 2004).

\subsection{Generalization for scattering multilayer systems}

Equation (22) is valid for multilayer systems, where scattering and absorption are negligible in or between different snow layers. In the present work, we solely concentrate on non-scattering and non-absorptive media for which all scattered energy returns from the bottom of the snowpack. For cases where scattering occurs at the snow surface, at layer boundaries or within snow layers, or where microwaveabsorbing layers are present, the location of the main scattering center is difficult to define and depends strongly on the scattering and absorption properties of the snowpack.

The scattering properties are given by the ratio of grain size to wavelength but also by the surface roughness and the dielectric contrast between neighboring layers. Scattering within the snowpack can occur, for example, in old metamorphic snow like depth hoar, in snow which contains ice layers and melt crusts as well as in deep snow on glaciers where the snow depth exceeds the penetration depth of microwaves.

In the following we briefly outline how Eq. (22) can be generalized to estimate the CPD when scattering of different layers needs to be included. For generalization, we define - possibly complex - amplitude scattering factors $\mu_{j}$ for each layer boundary. The scattering contribution of the first layer boundary, the air-snow interface, is given by $\mu_{0}$. The phasor $e^{i \phi_{1}}$ describing the CPD of the first layer contributes with the backscatter amplitude factor $\mu_{1}$ of the firstto-second layer boundary to the total phase difference. The reflection after the second layer accumulates the CPD of the first and second layer, so that the second phasor is given by $e^{i\left(\phi_{1}+\phi_{2}\right)}$ and so on. The total phase difference is then

$$
\begin{aligned}
\phi_{\mathrm{CPD}} & =\mu_{0}+\mu_{1} \cdot e^{i \phi_{1}}+\mu_{2} \cdot e^{i\left(\phi_{1}+\phi_{2}\right)}+\ldots \\
& =\sum_{j=0}^{N} \mu_{j} \prod_{k=0}^{j} e^{i \phi_{j}} \text { with } \phi_{0}=0 .
\end{aligned}
$$

Scattering within layers can be included by subdividing homogeneous layers into sufficient numbers of finite layers.

For homogeneously scattering and/or absorbing volumes, $\left|\mu_{j}\right|$ decreases exponentially, whereas $\mu_{j}$ can be quite variable for snow in, for example, the percolation zone of glaciers (Parrella et al., 2016) which contains melt crusts or ice layers. In such cases, assumptions must be made for the penetration depth or the penetration depth must be determined independently and the inversion of the CPD to determine the anisotropy can quickly be questionable.

\subsection{Contribution of a rough ground surface}

The anisotropy of snow can be determined from the CPD when the CPD of the underlying ground is 0 or at least known (cf. Sect. 5.6). Radar experiments have shown that the CPD is close to 0 for soil at small incidence angles but the CPD shows an increasing standard deviation for rough surfaces. It has also been found that the CPD increases to a few tens of degrees with increasing incidence angle for rough soil (Sarabandi, 1992; Oh et al., 2002). The CPD is also influenced by vegetation cover, especially for oriented vegetation (Ulaby et al., 1987). Therefore, CPD measurements of snow-free ground are advisable to verify whether any CPD bias exists.

\section{Experimental data}

For validation of our model we analyzed radar data acquired within the Nordic Snow Radar Experiment (NoSREx) campaigns (Lemmetyinen et al., 2013). The NoSREx campaigns consisted in extensive field measurements and various active and passive microwave measurements acquired at a test site near the town of Sodankylä in Northern Finland. The test site, shown in Fig. 5, is an almost flat forest clearing surrounded by boreal forest. The site is covered by low taiga-type vegetation on mineral soil. The site is equipped with various sensors to measure meteorological data and snow properties. For the radar measurements, two sectors were defined: sector 1 in the center of the forest clearing and sector 2 between trees.

\subsection{Microwave measurements}

The radar data were acquired by the SnowScat instrument (SSI) installed on the $9 \mathrm{~m}$ high tower shown in Fig. 5. The inset shows the SSI with its two horn antennas.

SnowScat is a fully polarimetric, coherent, continuouswave, stepped-frequency, real-aperture radar and operates between 9.2 and $17.8 \mathrm{GHz}$ (Wiesmann et al., 2008; Werner 


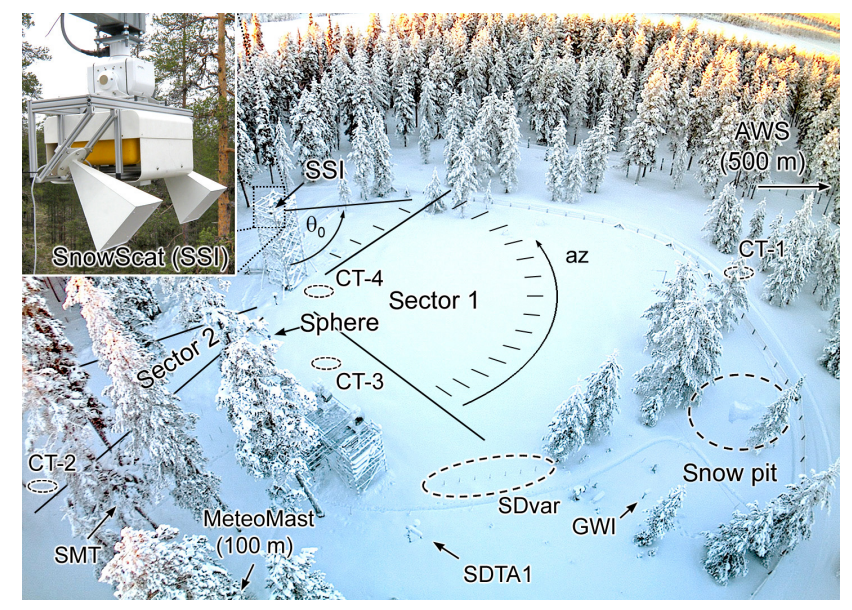

Figure 5. The radar data and meteorological measurements were acquired on the above shown test site near the town of Sodankylä, Finland. The SnowScat instrument (SSI; inset) was mounted on a $9 \mathrm{~m}$ high tower. The reference target (sphere) used for calibration can be located behind a tree. The SSI scanned sectors 1 and 2 with different azimuth (az) and incidence angles $\theta_{0}$. The meteorological sensors SMT, MeteoMast, SDAT1, GWI, and AWS are specified in Table 1. Snow profiles were taken at CT-1 to CT-4 for analysis by computer tomography. Snow density was measured in the snow pit, with the Gamma Water Instrument (GWI), and was also derived from the snow water equivalent (SWE) determined by the SSI as described in Leinss et al. (2015). The variability of snow depth was measured with seven sticks inside the ellipse "SDvar".

et al., 2010; Wiesmann and Werner, 2010). It was originally developed and built for snow backscatter measurements within the ESA ESTEC project KuScat (contract no. 42000 20716/07/NL/EL). Both horn antennas can transmit and receive in horizontal $(\mathrm{H})$ and vertical $(\mathrm{V})$ polarization. The $(-3 \mathrm{~dB})$ beam width of the antennas ranges from 5 to $12^{\circ}$, depending on polarization and frequency.

Radar acquisitions of the two sectors were acquired every four hours. The sectors were scanned in azimuth subsectors of $6^{\circ}$ by rotating the antennas around the vertical axis (az). The scan was done for each of the four nominal incidence angles $\left(\theta_{0}=30,40,50\right.$, and $\left.60^{\circ}\right)$, resulting in $17 \times 4$ acquisitions for sector 1 and $5 \times 4$ acquisitions for sector 2 . Each subsector was measured in all four polarization combinations, $\mathrm{VV}, \mathrm{HH}, \mathrm{VH}$, and $\mathrm{HV}$, using the full frequency range. A detailed description showing the acquisition geometry, the antenna patterns, and the polarimetric backscatter signal of both sectors can be found in Leinss et al. (2015); in this publication, the snow water equivalent (SWE), later used to estimate the snow density, was determined from SSI data by means of differential radar interferometry.

In the present work, we assume that during dry snow conditions all energy is backscattered from the ground below the snowpack. This assumption is justified because SWE could be precisely determined in Leinss et al. (2015), where a requirement for successful SWE measurements was a snow-
Table 1. Abbreviations for different sensors and measurements.

\begin{tabular}{|c|c|}
\hline SSI & SnowScat instrument (ground-based radar) \\
\hline sphere & Calibration target for the SSI \\
\hline CT-no. & $\begin{array}{l}\text { Snow profile number acquired in the field } \\
\text { and analyzed by computer tomography }(\mu \mathrm{CT})\end{array}$ \\
\hline CT-1 & Profile 1, sampling date: 3 March 2011 \\
\hline CT-2 & Profile 2, sampling date: 21 December 2011 \\
\hline CT-3 & Profile 3, sampling date: 1 March 2012 \\
\hline CT-4 & Profile 4, sampling date: 28 February 2013 \\
\hline SDAT1 & Snow depth and air temperature sensor no. 1 \\
\hline SMT & Sensors for soil moisture and soil temperature \\
\hline MeteoMast & $\begin{array}{l}\text { Meteorological mast (snow depth, } \\
\text { snow temperature profiles) }\end{array}$ \\
\hline SDvar & Snow depth variability course \\
\hline AWS & Automatic weather station \\
\hline GWI & $\begin{array}{l}\text { Gamma Water Instrument (SWE measurement } \\
\text { by gamma ray absorption) }\end{array}$ \\
\hline
\end{tabular}

pack transparent for microwaves. The assumption of a transparent snowpack is further supported by the analysis of the radargrams (Figs. 9 and 10 in Leinss et al., 2014a) which show a distinct range shift of $1.2 \mathrm{~m}$ of the antenna pattern at the onset of snow melt. This shift measured at $\theta_{0}=40^{\circ}$ is proportional to the slant range difference between the wet snow surface of $80 \mathrm{~cm}$ height, as observed in April 2012 and 2013, and the underlying ground which had been visible trough the snow pack during dry snow conditions before snow melt.

\subsection{Meteorological instruments, snow depth, and density determination}

Several meteorological sensors were installed on the test site at the locations shown in Fig. 5. Abbreviations for the sensors are listed in Table 1; technical details can be found in Kontu et al. (2011). Snow depth $(\mathrm{SD}=\Delta Z)$ and air temperature were measured by the sensor SDTA1. Soil temperature (two sensors at $2 \mathrm{~cm}$ depths) and soil moisture (four sensors, two at $2 \mathrm{~cm}$ and two at $10 \mathrm{~cm}$ depth) were measured by a sensor network named SMT. SD and temperature profiles within the snowpack were measured by the meteorological mast (MeteoMast) $100 \mathrm{~m}$ east of the SSI. An automatic weather station (AWS), located $500 \mathrm{~m}$ north of the SSI, measured SD, air temperature, and other meteorological parameters.

The variability of SD on the test site was measured with seven sticks located $1 \mathrm{~m}$ apart at the so-called "snow depth variability course" (SDvar). The seven sticks are located in the ellipse "SDvar" (Fig. 5). The SDvar measurements showed a quite homogeneous SD distribution with a standard deviation of $2-3 \mathrm{~cm}$ during dry snow conditions. This allows for comparison of snow data measured at different locations within the test site. 
The depth-averaged snow density $\rho_{\text {avg }}$ was manually measured in the snow pit once every week; it was also calculated from SD measured by SDTA1 and from SWE measurements (SWE in millimeter water column equivalent $\left.=\Delta Z \cdot \rho_{\text {avg. }} / \rho_{\text {ice }}\right)$. SWE was obtained from the SSI during dry snow conditions (Leinss et al., 2015) and from gamma ray absorption measurements of the snowpack by the Gamma Water Instrument (GWI) during wet snow conditions. A short description of the GWI is given in Kontu et al. (2011) and Leinss et al. (2015).

\subsection{Computer tomography profiles}

The microstructure of four vertical snow profiles, CT1 ... CT-4, sampled in the field on the dates given in Table 1, was determined using computer tomography $(\mu \mathrm{CT})$. The locations of the profiles are shown in Fig. 5. For each profile, vertically overlapping samples of about $10 \mathrm{~cm}$ height were taken to cover entire snow depth profiles. For later analysis by means of $\mu \mathrm{CT}$, the snow samples were cast for transportation from Finland to the cold lab at the WSL Institute for Snow and Avalanche Research SLF in Switzerland. An analysis of the $\mu \mathrm{CT}$ data, here used to determine the anisotropy, was already published with respect to other snow structure parameters in Proksch et al. (2015). Here we briefly summarize the casting and processing methodology.

The samples were cast using diethyl phthalate (DEP) to preserve the snow microstructure. The casting procedure and an accuracy analysis of cast and non-cast samples are described in Heggli et al. (2009). In the cold lab, the samples were scanned with a nominal resolution (voxel size) ranging from $10 \mu \mathrm{m}$ for new snow to $20 \mu \mathrm{m}$ for depth hoar. The size of the evaluated volumes ranged from $67 \mathrm{~mm}^{3}$ for CT-1, CT-2, and CT-3 $(512 \times 512 \times 256$ voxel with $10 \mu \mathrm{m}$ voxel size $)$ to $917 \mathrm{~mm}^{3}$ for CT-4 $(512 \times 512 \times 600$ voxel with $18 \mu \mathrm{m}$ voxel size). The resulting 3-D gray-scale images were filtered using a Gaussian filter ( $\sigma=1$ voxel; filter kernel support $=2$ voxel) and were then segmented into binary images. For snow-air segmentation, the intensity threshold was chosen at the minimum between the DEP peak and the air peak in the histograms of the gray-scale images.

\subsection{SnowScat data processing and CPD calibration}

The SSI raw data measured in frequency domain for each azimuth and incidence angle $\left(\mathrm{az}, \theta_{0}\right)$ were windowed to select a specific frequency band of $2 \mathrm{GHz}$ bandwidth. The selected bandwidth was then focused in range $r$ to the single-lookcomplex (SLC) format (for procession details see Leinss et al., 2015). The pixels of an SLC image of polarization "pol" represent the complex-valued backscatter coefficients $S_{\mathrm{pol}}\left(r, \theta_{0}, \mathrm{az}\right)$. The copolar coherence (Eq. 10) for each az and $\theta_{0}$ was then obtained from the backscatter coefficients $S_{\mathrm{VV}}$ and $S_{\mathrm{HH}}$. The ensemble averages $\langle\cdot\rangle_{r}$ contained about 150-300 range pixels covering the full width $(-3 \mathrm{~dB})$ of the antenna footprint. By summing over the antenna footprint, slightly different $\mathrm{CPD}$ values have been averaged due to the incidence angle variation of $5-8^{\circ}$ within the common antenna footprint of both polarizations. Still, across the footprint, the incidence angle dependence of the CPD is sufficiently linear so that no systematic errors are expected. For noise and speckle reduction, the copolar coherences of different az subsectors with the same incidence angle were averaged before the CPD was calculated. The obtained CPD showed some temporal drifts; therefore a metallic sphere was used for calibration (see Appendix D).

\subsection{Selecting valid acquisitions}

Invalid acquisitions were removed before the analysis with the help of the calibration data. Acquisitions were classified as invalid when the CPD or the radar cross section of the reference targets (sphere, plate) deviated too far from the expected values or when the temporal trend of the sphere and the plate were not in agreement. In the two seasons before 18 November 2011, when the plate target was not installed yet, the sphere showed very stable results; therefore the data were considered as valid. For sector 2, which was located between trees, some subsectors on the left- and right-hand side were disturbed by trees (Leinss et al., 2015, Fig. 3) and were therefore excluded from the analysis.

\subsection{Measurements: 4 years of CPD time series}

Four years of CPD time series, acquired by SnowScat between 2009 and 2013, are plotted in Figs. 6-9 together with meteorological measurements. Dark gray shading in April and May indicates snow melt. Snow-free conditions are indicated by light gray shading in fall and May/June.

The upper three panels show meteorological parameters (for abbreviations and locations see Table 1 and Fig. 5). Dashed lines show snow depths measured by the three sensors SDAT1, AWS, and MeteoMast. Errors bars indicate the mean and standard deviation from the SD-variability course "SDvar". The solid blue line shows the average snow depth of SDAT1, AWS, and MeteoMast. Air and soil temperatures from SDAT1 and SMT are plotted below the snow depth. The third panel shows soil moisture from two locations, each at 2 and $10 \mathrm{~cm}$ depth, measured by the sensors of SMT (brown). Snow density (solid black line) was determined from snow depth measurements (SDAT1) and from SWE as described in Sect. 4.2. Manual density measurements from the snow pit are shown as black dots.

The lower three panels show the CPD $\left(=\phi_{\mathrm{VV}}-\phi_{\mathrm{HH}}\right)$ for different incidence angles $\theta_{0}$ (fourth panel) and frequencies (fifth panel), as well as the co-polar coherence $\gamma_{\mathrm{VV}, \mathrm{HH}}$ for different frequencies at the largest incidence angle $\theta_{0}=60^{\circ}$, where it is most sensitive to volume scattering.

In the following paragraphs we summarize the main characteristics observed during the experiment. Common for all 


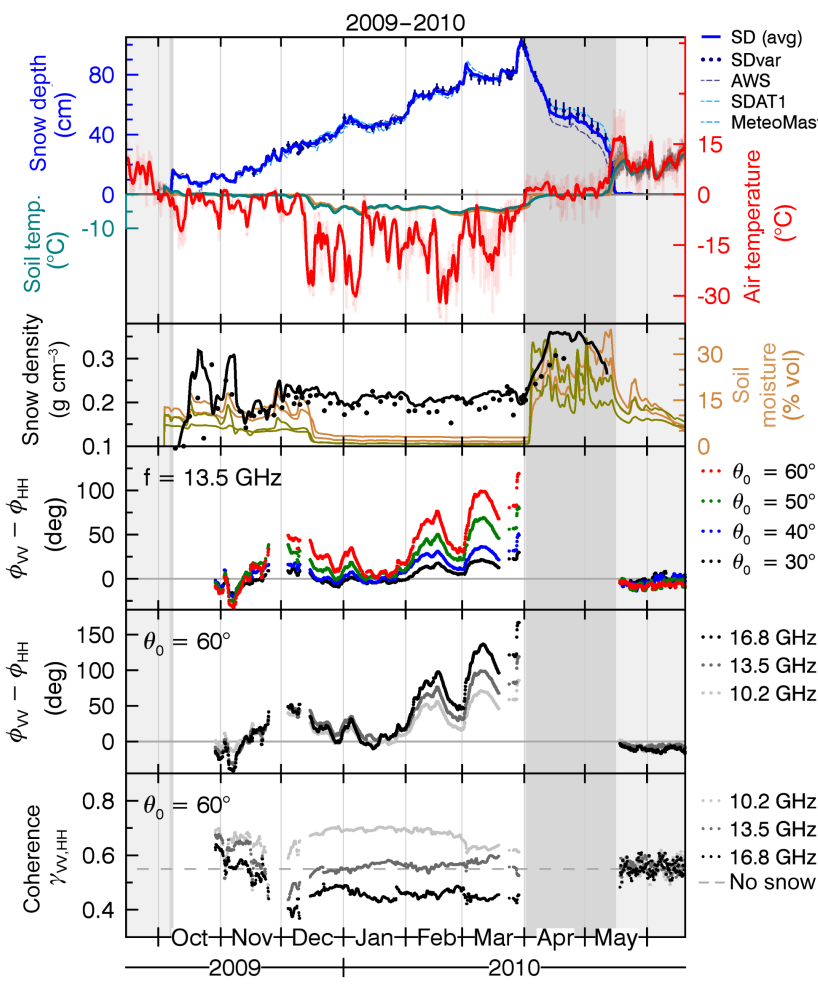

Figure 6. Winter season 2009-2010. Top panels: meteorological data measured by the sensors described in Sect. 4.6. Bottom panels: CPD and copolar coherence measured by the SSI for different incidence angles and frequencies. The dark gray shading shows snow melt.

four seasons is a rising CPD during snowfall, although the maximum CPD is observed a few days after snowfall. The CPD decreases during cold periods with temperatures well below $0{ }^{\circ} \mathrm{C}$ for which precipitation is weak. During snow melt, the CPD is close to 0 because microwave penetration into the wet snowpack is inhibited. Soil moisture correlates well with snow melt but does not affect the CPD, even for non-frozen soil in early winter.

The copolar coherence $\gamma \mathrm{VV}$,HH ranges from 0.4 to 0.7 during dry snow conditions, with lower values for higher frequencies. At $16.8 \mathrm{GHz}$ at $\theta_{0}=60^{\circ}$ the coherence is lower during winter $(\approx 0.4)$ compared to snow-free conditions $\left(\gamma_{\mathrm{VV}, \mathrm{HH}} \approx 0.55\right.$, horizontal dashed line "no snow"), which indicates some weak scattering in the snow volume. The highest values $\gamma \mathrm{VV}, \mathrm{HH}=0.7 \ldots 0.8$ were measured during snow melt, where microwave penetration depth is very small (a few centimeters) and scattering occurs at the snow surface. After all snow has melted, the coherence decreased to $\approx 0.5 \ldots 0.6$ due to some volume scattering at the low vegetation.

\subsection{CPD interpretation with respect to snow conditions}

The four analyzed winter seasons showed quite different snow conditions. In the following, we provide an interpre-

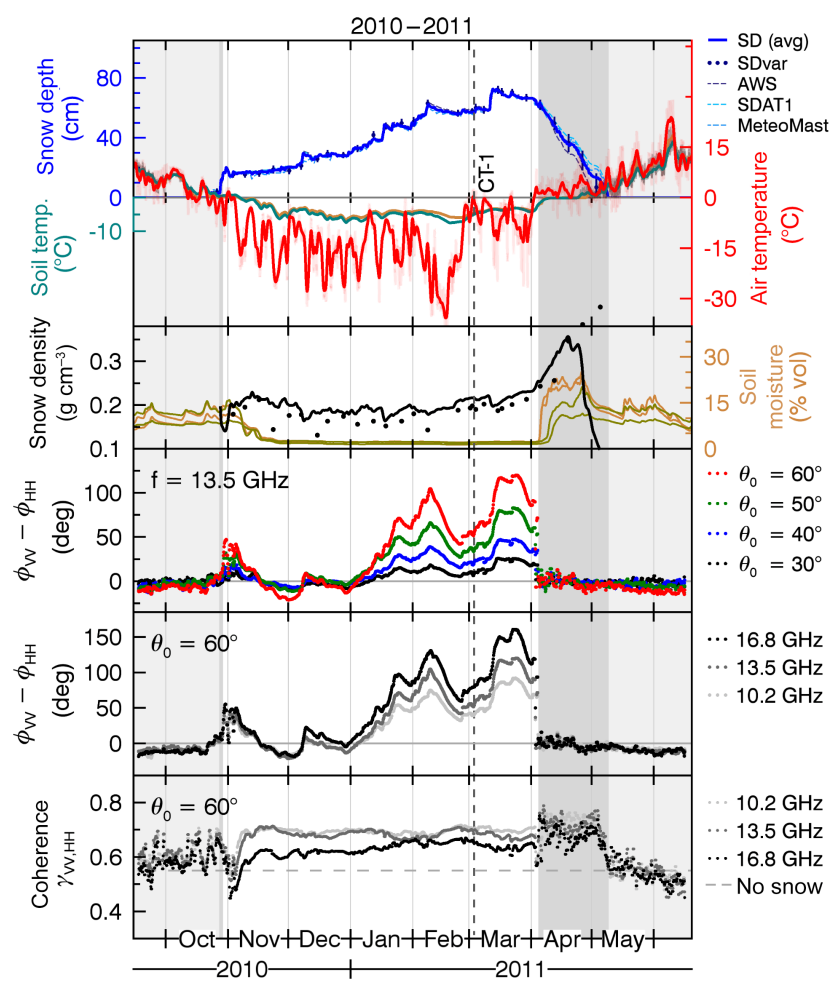

Figure 7. Winter season 2010-2011. Meteorological and radar data as shown in Fig. 6 and described in Sect. 4.6. The vertical dashed line shows the date when the snow profile CT-1 was acquired.

tation of the measured CPD time series with respect to snow properties which were observed in the field and which were documented in Lemmetyinen et al. (2013, p. 425/49).

The winter of 2009-2010 was characterized by mild temperatures until mid-December which caused delayed soil freezing compared to average years. Snow accumulated gradually (causing a small positive CPD) and the mild temperatures lead to larger snow densities of $0.2 \mathrm{~g} \mathrm{~cm}^{-3}$ in early winter compared to other years. Due to warm temperatures, depth hoar was largely absent and melt-refreeze events in early December caused the formation of a crust in the shallow snowpack, which was later covered by snow. Later in winter, two major snowfall events occurred. The first happened during early February, after which the CPD increased by more than $50^{\circ}$ but decreased quickly due to strong temperature gradients causing a fast metamorphism into vertical structures. The second major snowfall occurred during the night from 2 to 3 March 2010, when a fast rise in temperatures together with $20 \mathrm{~mm}$ precipitation caused some snow settling. Despite additional fresh snow of low density, a slight increase in the average snow density can be observed in Fig. 6. The settling caused an abrupt increase of the CPD of about $+20^{\circ}$ during the night, followed by a total increase of more than $+50^{\circ}$ within the 5 following days. Snow settling and collapse of weak layers are discussed with respect to the SnowScat anomaly on 2-3 March 2010 in Lemmetyi- 


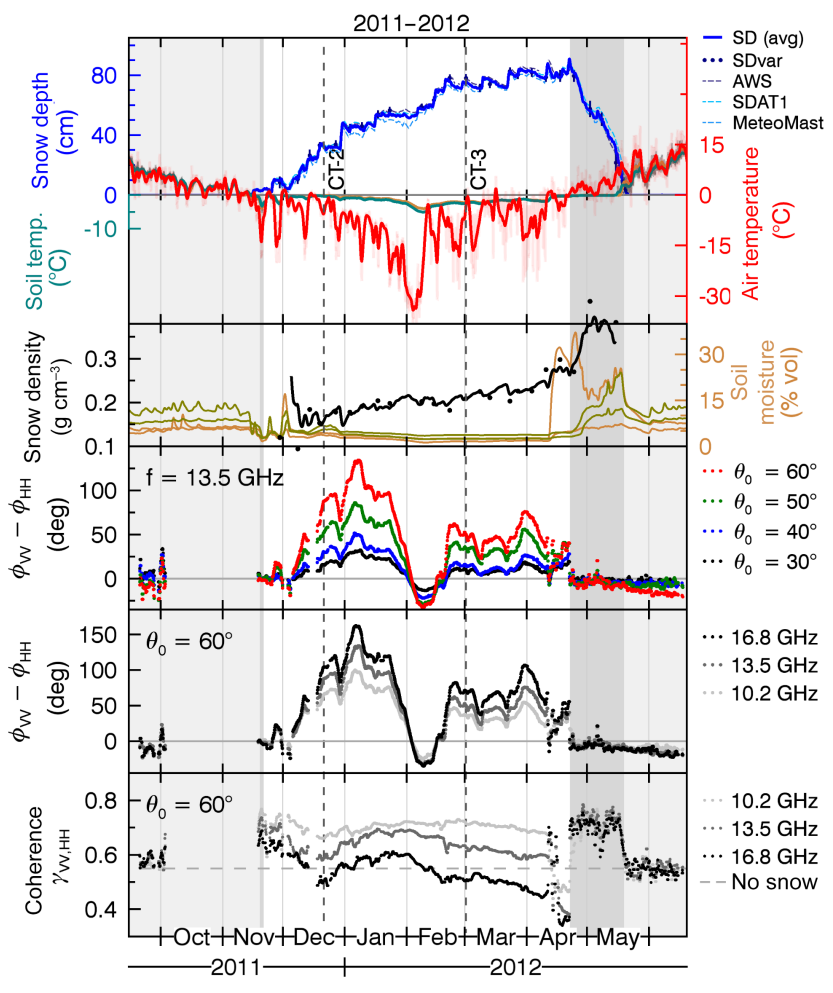

Figure 8. Winter season 2011-2012. Meteorological and radar data as shown in Fig. 6 and described in Sect. 4.6. Two vertical dashed lines shows the date when the profiles CT-2 and CT- 3 were acquired.

nen et al. (2013, 214-240). Our observations support their arguments, as a strong increase of the CPD is related to fresh snow, snow settling, and a possible collapse of weak layers with vertical structures.

The winter of 2010-2011 was characterized by very cold temperatures and a relatively thin snow cover. The strong temperature gradients led to a distinct layer of depth hoar. The slightly negative CPD in December indicates a weak anisotropy with vertical structures in the snowpack. From January until March, the CPD increased with snowfall but was disrupted by a period of very cold temperatures in February, during which the CPD decreased by $-50^{\circ}$.

The winter of 2011-2012 was characterized by initially exceptionally mild temperatures and late but intense snowfall during December. The weak temperature gradient from mid-December until mid-January caused almost no metamorphism into vertical structures. Therefore, a thick layer of horizontally oriented, settled fresh snow was preserved and a maximum CPD of $+135^{\circ}$ was observed at 7 January, 9 days after $20 \mathrm{~cm}$ of fresh snow fell. Almost no depth hoar was observed due to the insulating effect of the thick snowpack. The extremely large phase differences disappeared relatively quickly during very cold air temperatures between -15 and $-35^{\circ} \mathrm{C}$ in the second half of January until mid-February, when the CPD even changed sign, so that a minimum CPD of $-30^{\circ}$ was observed on 9 February. After various snow-

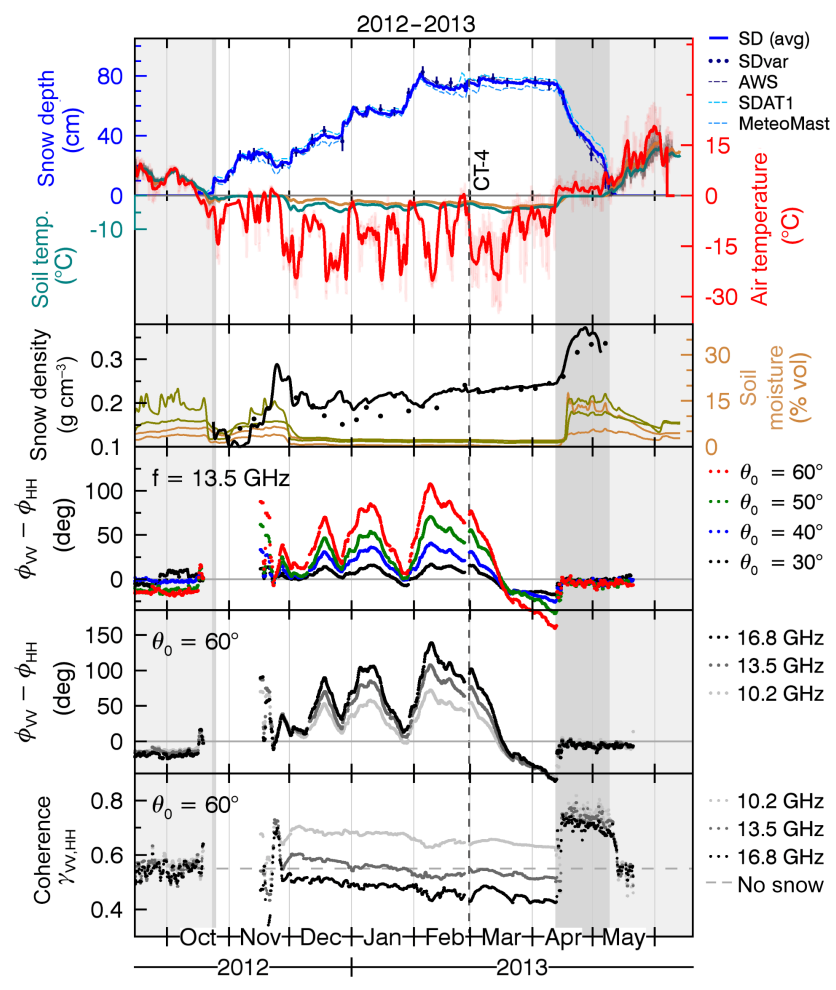

Figure 9. Winter season 2012-2013. Meteorological and radar data as shown in Fig. 6 and described in Sect. 4.6. The vertical dashed line shows the date when the snow profile CT- 4 was acquired.

fall events, the negative phase differences disappeared. On 12 April, the snow surface melted and refroze afterwards. A significant drop of the copolar coherence below values observed during snow-free conditions (Fig. 8, lowest panel) indicates increased volume scattering or even residual melt water in the snowpack. A change in the backscatter pattern observed in Leinss et al. (2014a, Fig. 9) supports the observation of increased volume scattering. During the time around 12 April, when the snow surface was wet, the CPD dropped for a few days to 0 but recovered afterwards during a short period of negative temperatures before snow melt.

The winter of 2012-2013 was again characterized by very mild temperatures but early and heavy snowfall during November, followed by three additional major snowfall events, which caused a very clear peak-like signal in the CPD. The peaks appear a few days after snowfall ended, which indicates that settling of fresh snow is responsible for an increase of the CPD. In February, after the last heavy snowfall, a CPD of more than $+100^{\circ}$ was reached. From March until mid-April, no snowfall occurred and low temperatures caused a strong metamorphism for a period of 6 weeks, after which a minimum CPD of $-60^{\circ}$ was observed. With the onset of snow melt, the CPD jumped to 0 due wet snow and the resulting small microwave penetration depth. 


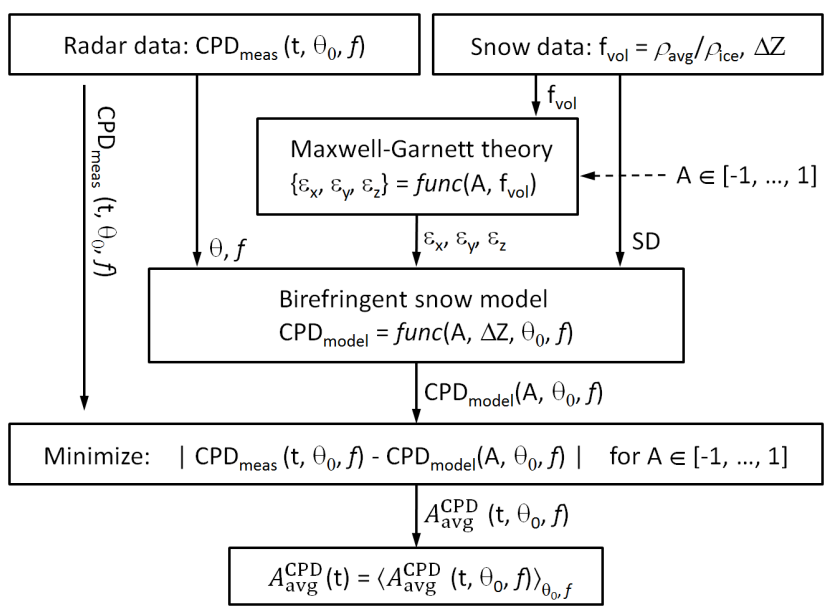

Figure 10. Processing chain used to estimate the average anisotropy of the snowpack, $A_{\mathrm{avg}}^{\mathrm{CPD}}$. The anisotropy can be estimated from the measured CPD, by minimizing the difference between modeled and measured data with respect to $A$ when snow depth $\Delta Z$ and the ice volume fraction $f_{\mathrm{vol}}$ are known. The anisotropy $A_{\mathrm{avg}}^{\mathrm{CPD}}\left(t, \theta_{0}, f\right)$ was calculated independently for all incidence angles, $\theta_{0}$, and frequencies, $f$, and the results were averaged to obtain $A_{\mathrm{avg}}^{\mathrm{CPD}}(t)$.

\section{Analysis}

\subsection{Estimation of the average structural anisotropy}

The developed electromagnetic model in Sects. 2 and 3 is free of fit parameters. Therefore, the copolar phase difference, measured and averaged over all azimuth subsectors, $\mathrm{CPD}_{\text {meas. }}$, can be inverted with the additional information of snow depth and a good approximation of snow density (as discussed at the end of Sect. 3.4) to get a CPD-based estimate for the depth-average anisotropy, $A_{\mathrm{avg}}^{\mathrm{CPD}}\left(t, \theta_{0}, f\right)$.

For the analysis, we assumed that the snowpack consisted of a single layer of constant anisotropy. We further assumed that the snow properties (depth, density, anisotropy, and also scattering properties of the underlying soil) do not vary spatially across the test site so that we can compare measurements done with different incidence angles and with different antenna footprints. The measurements of the snow depth variability course, SDvar, and the careful preparation of the test site's surface support these assumptions. The area observed by the SSI covers the center of the forest clearing such that variations of snow properties due to a proximity to trees should be negligible (this is only true for sector 1 , not for sector 2 located between trees). A variable snow depth due to wind drift is unlikely, as the test site is sufficiently wind protected by the surrounding trees (Fig. 5).

The anisotropy $A_{\mathrm{avg}}^{\mathrm{CPD}}$ is estimated from Eq. (22) using $\mathrm{CPD}_{\text {meas. }}\left(t, \theta_{0}, f\right)$ with the radar parameters microwave frequency $f$ and incidence angle $\theta_{0}$. The block diagram (Fig. 10) shows a sketch of the processing chain to determine $A_{\mathrm{avg}}^{\mathrm{CPD}}$. The required in situ measured parameters snow depth $\Delta Z$ (from SDAT1) and the depth-averaged snow density $\rho_{\text {avg }}$ are shown in Figs. 6-9. The ice volume fraction $f_{\mathrm{vol}}=\rho_{\mathrm{avg}} / \rho_{\text {ice }}$ follows from snow density. For every measurement time $t$, the depth-averaged CPD-based estimate $A_{\mathrm{avg}}^{\mathrm{CPD}}\left(t, \theta_{0}, f\right)$ follows by minimization of the difference

$$
\left|\mathrm{CPD}_{\text {meas }}\left(t, \theta_{0}, f\right)-\mathrm{CPD}_{\text {model }}\left(A\left(\theta_{0}, f, t\right), \theta_{0}, f\right)\right|,
$$

with respect to $A \in[-1,1]$. CPD time series at 16 different frequencies between 10 and $17 \mathrm{GHz}$ and at four different incidence angles were evaluated in Eq. (25); a few of them are shown in Figs. 6-9. Anisotropy values of all 16 frequencies but only from the three larger incidence angles $\theta_{0}=40$, 50 , and $60^{\circ}$ were later averaged to determine $A_{\mathrm{avg}}^{\mathrm{CPD}}(t)$, since the CPD measurements with the smallest incidence angle $\left(\theta_{0}=30^{\circ}\right)$ showed the highest sensitivity to calibration errors. $A_{\mathrm{avg}}^{\mathrm{CPD}}(t)$ was therefore determined from $48(=16 \times 3)$ independent estimates $A_{\mathrm{avg}}^{\mathrm{CPD}}\left(t, \theta_{0}, f\right)$. The standard deviation for each time $t$ is determined by the distribution of all 64 $(=16 \times 4)$ estimates, $A_{\mathrm{avg}}^{\mathrm{CPD}}\left(t, \theta_{0}, f\right)$, around their average $A_{\mathrm{avg}}^{\mathrm{CPD}}(t)$. The average standard deviation $\sigma_{\text {Aavg }}^{\mathrm{CPD}} \approx 0.005$ is well below the obtained anisotropy range between -0.05 and +0.2 . The standard deviation varies with snow depth and is shown as a gray bar below the anisotropy in Fig. 11.

The time series in Fig. 11 show that the largest positive anisotropy $A_{\mathrm{avg}}^{\mathrm{CPD}} \approx+0.2$ was found for December 2011 after intense snowfall and while temperature gradient metamorphism was very weak. The largest negative anisotropies were found for November $2010\left(A_{\mathrm{avg}}^{\mathrm{CPD}} \approx-0.06\right)$ where strong temperature gradients in the thin snowpack were present. Large negative anisotropies were also found in February $2012\left(A_{\mathrm{avg}}^{\mathrm{CPD}} \approx-0.05\right)$ and April $2013\left(A_{\mathrm{avg}}^{\mathrm{CPD}} \approx-0.05\right)$ after periods of very cold temperatures without precipitation.

The structural and dielectric anisotropy (Fig. 11) seems small compared to reported literature results. However, the snow profile analysis (Fig. 16 in Sect. 5.3) reveals that the anisotropy $A_{\mathrm{avg}}^{\mathrm{CPD}}$ averages much larger positive and negative anisotropies, resulting from horizontal structures at the snow surface and vertical structures in older layers.

While discussing the structural anisotropy of snow derived from dielectric anisotropy it seems relevant to recall that single ice crystals also show a birefringence. Fujita et al. (2014) reported that the dielectric anisotropy due to oriented crystal fabrics is often much lower than dielectric anisotropy expected from a structural anisotropy. In Appendix A we used measurements of the crystal orientation fabric (COF) in snow from Riche et al. (2013) to estimate a maximum dielectric anisotropy of $\Delta \varepsilon=-0.002$, corresponding to a structural anisotropy of $A=-0.02$. This is small compared to the measurements shown in Fig. 11 and confirms the statement of Fujita et al. (2014). It is worth noting here that the dielectric anisotropy due to the vertical crystal orientation of fresh snow has the opposite sign as the dielectric anisotropy due to the horizontal structural orientation of fresh snow. 


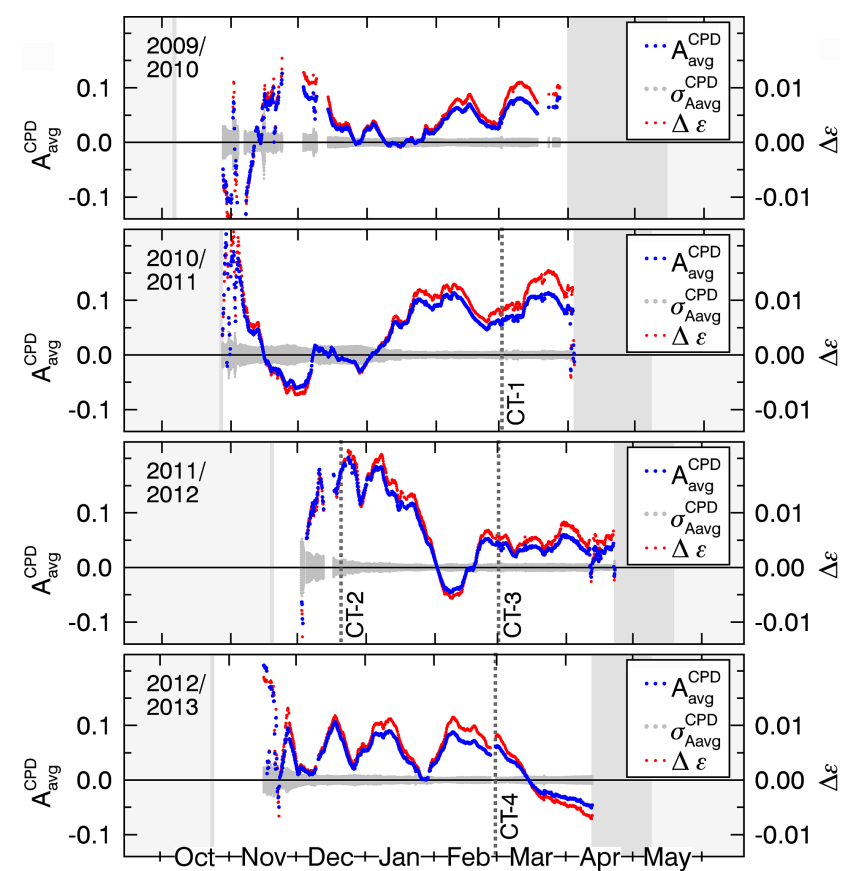

Figure 11. Average anisotropy of the snowpack, $A_{\mathrm{avg}}^{\mathrm{CPD}}$, determined during dry snow conditions for the winter seasons from 2009 to 2013. The anisotropy was derived from the CPD measured by the SnowScat instrument. The standard deviation $\sigma_{\text {Aavg }}^{\mathrm{CPD}} A_{\mathrm{avg}}^{\mathrm{CPD}}$, calculated from measurements at different frequencies and incidence angles, is shown as the time-varying gray bar below the anisotropy. The dielectric anisotropy $\Delta \varepsilon=\varepsilon_{x}-\varepsilon_{z}$ is shown in red. The dark gray shading in April/May indicates the period of snow melt; the light gray shadings (October/November, May/June) indicate snowfree conditions. The four dashed vertical lines show the times when the anisotropy was measured by computer tomography (CT-1, -2, -3 , and -4$)$.

\subsection{Incidence angle and frequency dependence}

The larger the incidence angle, the better the alignment of the vertically polarized microwaves with the optical axis of birefringent snowpack. The CPD must therefore increase with increasing incidence angle. This has already been observed in the CPD time series plotted for different incidence angles in the middle panels of Figs. 6-9.

The electromagnetic model of Sect. 3 predicts a nonlinear incidence angle dependence due to refraction in the snowpack (Fig. 3, left panel). To verify the nonlinear incidence angle dependence, we selected five dates spread over the four winter seasons to cover the maximum available range of CPDs. For each date we used measured snow density $\rho_{\text {avg }}$ and snow depth $\Delta Z$ together with the averaged CPD-based anisotropy, $A_{\mathrm{avg}}^{\mathrm{CPD}}$, to model the expected incidence angle dependence. A comparison of modeled and measured CPDs is shown in Fig. 12 (left panel) for the five selected dates.

The CPD is modeled to be proportional to the depth of a snowpack which is transparent for microwaves. The deeper
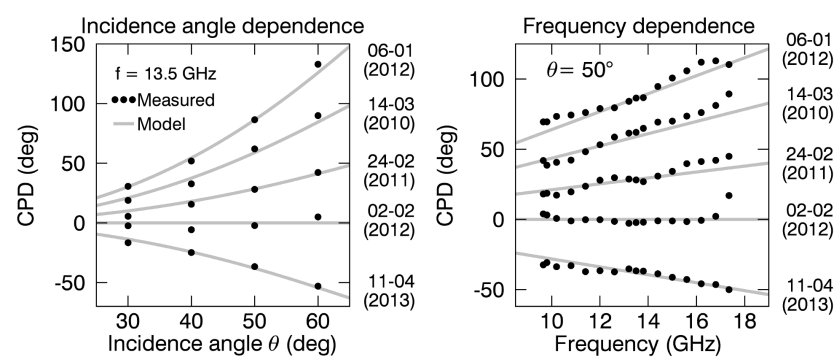

Figure 12. Left panel: incidence angle dependence of measured CPD vs. modeled incidence angle dependence. Right panel: frequency dependence of measured CPD vs. modeled linear frequency dependence.

the snow and the higher the frequency, the more wavelengths "fit" in the propagation path length through the snow volume and the higher the expected phase difference. This linear frequency dependence is described by Eq. (22), which is proportional to $\lambda^{-1}$. Larger CPD values were indeed measured for higher frequencies $(f=10.2,13.5$, and $16.8 \mathrm{GHz})$ as shown in the second-last panel of Figs. 6-9. For a more quantitative insight, we plotted the CPD measured for 16 different frequencies in Fig. 12 (right panels). The CPD was plotted for the same five dates as in Fig. 12 (left panels). As expected, the CPD shows approximately a linear dependence on frequency.

In order to get a better quantitative measure of how well the electromagnetic model fits to measured data, we did a statistical analysis and compared the modeled phase difference, $\mathrm{CPD}_{\text {model }}\left(A_{\mathrm{avg}}^{\mathrm{CPD}}(t), \theta_{0}, f\right)$, according to Eq. (22), with the measured phase difference, $\mathrm{CPD}_{\text {meas. }}\left(t, \theta_{0}, f\right)$. The mean deviation, as well as the standard deviation of $\mathrm{CPD}_{\text {model }}-\mathrm{CPD}_{\text {meas. }}$, was calculated over all acquisitions acquired during dry snow conditions separately for each incidence angle $\theta_{0}$ and for each frequency $f$.

The mean deviation is plotted over frequency and for each incidence angle in Fig. 13. The error bars indicate the standard deviation. The mean deviation is about $\pm 4^{\circ}$ (black dots in Fig. 13) and is almost always within the standard deviation (error bars). We measure larger deviation up to $+8^{\circ}$ only for $\theta=60^{\circ}$ and $f>14 \mathrm{GHz}$. Figure 13 shows that neither large deviations from the expected incidence angle dependence nor large deviations from the linear frequency dependence were found. The deviations of $\mathrm{CPD}_{\text {meas }}$ from the $\mathrm{CPD}_{\text {model }}$ are within the estimated calibration accuracy of $\pm 15^{\circ}$.

As measured and modeled data agree within a few degree, we conclude that our electromagnetic model is able to explain the observed CPD by considering snow as an optically anisotropic medium. The linear dependence on frequency confirms our assumption that the CPD is a volumetric property of snow. 


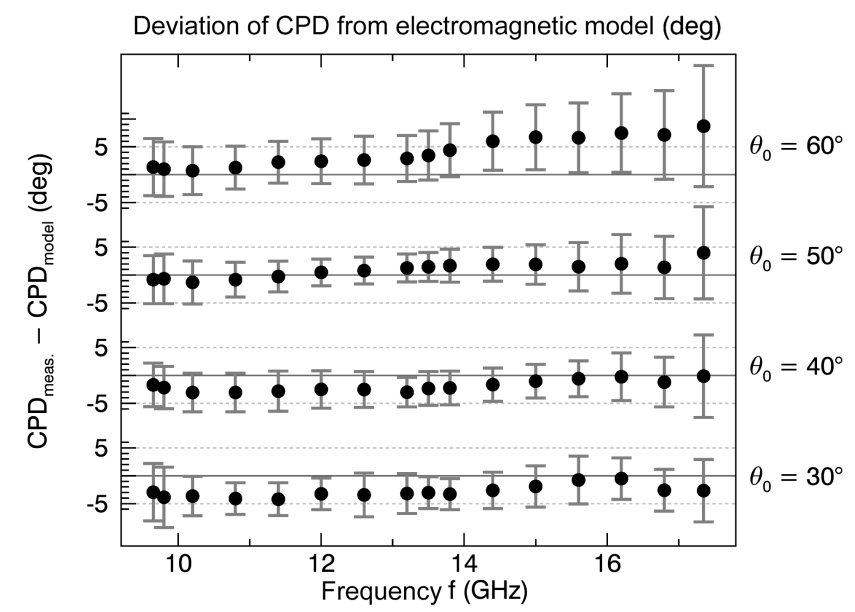

Figure 13. Deviation of measured and modeled CPD for different frequencies $f$ and different incidence angles $\theta_{0}$. Dots show the mean deviation $\mathrm{CPD}_{\text {meas. }}-\mathrm{CPD}_{\text {modeled }}$ of all data acquired during dry snow conditions. The error bars are the standard deviations calculated from about 5600 measurements acquired at different times.

\subsection{Validation with $\mu \mathrm{CT}$}

For validation the CPD-based estimates $A_{\text {avg }}^{\mathrm{CPD}}\left(t_{i}\right)$ were compared to $\mu \mathrm{CT}$-based estimates $A_{\mathrm{avg}}^{\mathrm{CT}}\left(t_{i}\right)$ obtained from in situ snow measurements. The four dates $t_{i}$, when the samples for $\mu \mathrm{CT}$-analysis were taken from the four snow pits, CT1 ... CT-4, are indicated as dashed vertical lines in Figs. 7-9 as well as in Fig. 11. Two examples of the 3-D images obtained by CT are shown in Figs. 14 and 15.

To obtain the anisotropy from the $\mu \mathrm{CT}$ data, the binary 3-D images were analyzed by means of spatial correlation functions according to Löwe et al. (2011). Exponential correlation lengths, $p_{\mathrm{ex}, x}, p_{\mathrm{ex}, y}$, and $p_{\mathrm{ex}, z}$, were derived from the correlation functions as described by Mätzler (2002). The anisotropy determined by tomography, $A_{\mathrm{CT}}$, is defined analogue to Eq. (1). Due to the symmetry in the $x$ and $y$ direction, $p_{\mathrm{ex}, x}$ and $p_{\mathrm{ex}, y}$ were averaged:

$A_{\mathrm{CT}}=\frac{\left(p_{\mathrm{ex}, x}+p_{\mathrm{ex}, y}\right)-2 p_{\mathrm{ex}, z}}{\left[\frac{1}{2}\left(p_{\mathrm{ex}, x}+p_{\mathrm{ex}, y}\right)+p_{\mathrm{ex}, z}\right]}$.

The anisotropy was determined for the entire snow profile with a vertical resolution of $1-2 \mathrm{~mm}$, depending on snow grain size. The obtained anisotropy profiles are shown in Fig. 16. For comparison, we added horizontal lines, which show the average anisotropy, $A_{\text {avg }}^{\mathrm{CT}}$, determined from CT and the average anisotropy, $A_{\mathrm{avg}}^{\mathrm{CPD}}$, determined from the CPD.

The first profile shown in Fig. 16 (CT-1) shows a slightly larger anisotropy, $A_{\mathrm{avg}}^{\mathrm{CPD}}=0.05$, compared to the average anisotropy derived from the $\mathrm{CT}$ data, $A_{\mathrm{avg}}^{\mathrm{CT}}=0.023$. For the profile CT- 1 only a limited number of data points were available with missing data from the lowest $10 \mathrm{~cm}$. However,
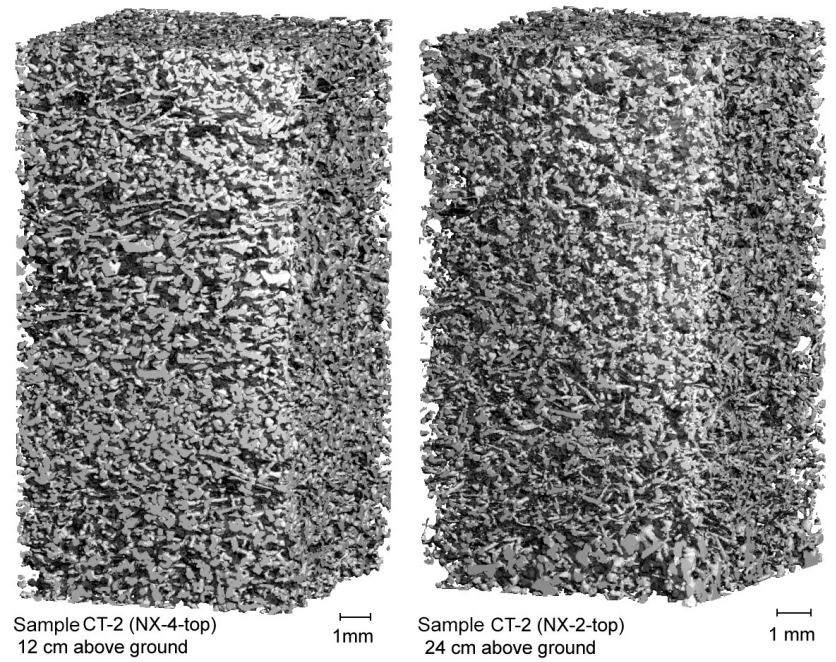

Figure 14. Two samples from the profile CT-2 (21 December 2011) taken $12 \mathrm{~cm}$ (left panel) and $24 \mathrm{~cm}$ (right panel) above ground. Horizontal structures are clearly visible in the left image but can also be identified in the right image. The CT-based average anisotropy of the two samples are $A_{\mathrm{avg}}^{\mathrm{CT}}=+0.26$ (left panel) and +0.16 (right panel). The vertically resolved anisotropy, $A_{\mathrm{CT}}$, determined every $2 \mathrm{~mm}$ depth by means of $\mu \mathrm{CT}$, is plotted in Fig. $16 \mathrm{~b}$ for both samples as blue dots.
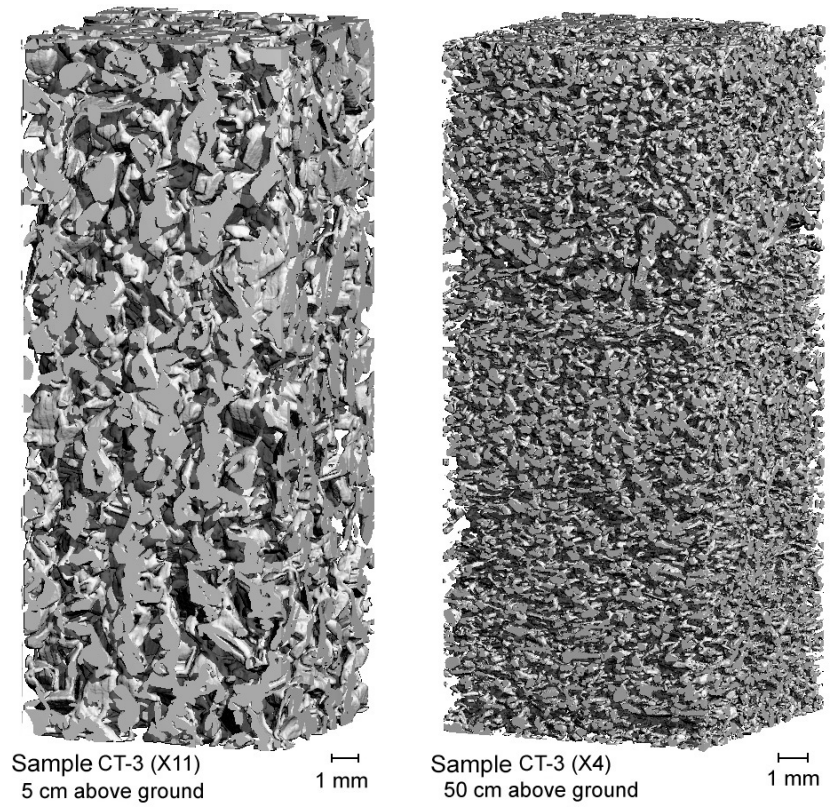

Figure 15. Two samples from the profile CT-3 (1 March 2012). The left profile, taken $5 \mathrm{~cm}$ above ground, shows old metamorphic snow (depth hoar) with vertical structures $\left(A_{\mathrm{avg}}^{\mathrm{CT}}=-0.24\right)$. The profile on the right, taken $50 \mathrm{~cm}$ above ground, shows horizontal structures $\left(A_{\mathrm{avg}}^{\mathrm{CT}}=+0.35\right)$ of fresh, settled snow which fell 2 weeks before the sample was taken. The vertically resolved anisotropy, $A_{\mathrm{CT}}$, determined every $2-5 \mathrm{~mm}$ depth by means of $\mu \mathrm{CT}$, is plotted in Fig. $16 \mathrm{c}$ for both samples as blue dots. 

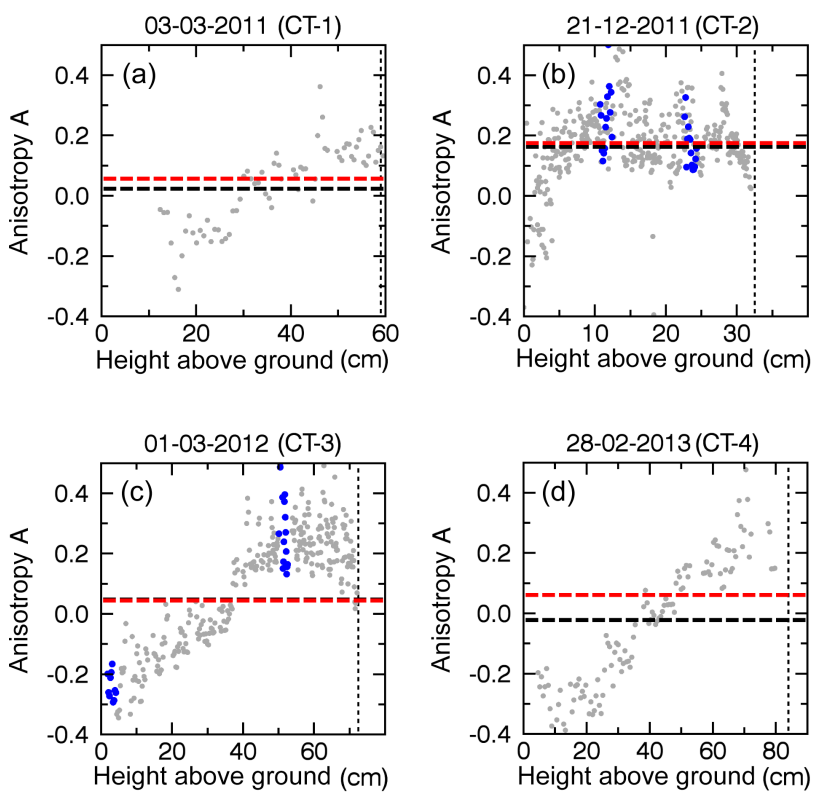

$$
\cdots A_{C T}=-A_{\text {avg }}^{C T}=-A_{\text {avg }}^{C P D} \ldots \text { snow depth } \cdots A_{C T}(\text { Figs.15+16) }
$$

Figure 16. Vertical profiles of the anisotropy, $A_{\mathrm{CT}}$, determined from computer tomography. For comparison, we plotted in each graph horizontal lines which show the depth-averaged anisotropies, $A_{\mathrm{avg}}^{\mathrm{CT}}$, together with the average anisotropy, $A_{\mathrm{avg}}^{\mathrm{CPD}}$, determined from the radar data (Fig. 11). (a) The profile CT-1 shows $30 \mathrm{~cm}$ of old snow with vertical structures from November/December 2010, which is covered by metamorphic snow that fell from mid-January to early February 2011 (see Fig. 7). (b) The profile CT-2 shows homogeneously distributed positive anisotropies which result from heavy snowfall during mild temperatures in December 2011 (see Fig. 8). (c) The profile CT-3 shows a thick layer with vertical structures of metamorphic snow in the lower $40 \mathrm{~cm}$ of the snowpack. In the upper $40 \mathrm{~cm}$ horizontal structures are visible which result from fresh snowfall mid-February 2012 (see Fig. 8). (d) Alternating snowfall and cold temperatures leads to an almost linearly increasing anisotropy in the profile CT-4 from late February 2013 (see Fig. 9).

depth hoar was observed in field observations for the bottom $10 \mathrm{~cm}$, indicating that $A_{\text {avg }}^{\mathrm{CT}}$ should even be smaller.

For the second and third profiles, CT-2 and CT-3, many CT data points were available and the difference in anisotropy is remarkably small and agrees within values of +0.008 and -0.004 , or +4 and $-8 \%$ relative to the anisotropy measured by $\mathrm{CT}$ of $A_{\mathrm{avg}}^{\mathrm{CT}}=+0.16$ and +0.05 .

For the fourth profile, CT- 4 , a larger difference of +0.08 was observed $\left(A_{\mathrm{avg}}^{\mathrm{CT}}=-0.02\right.$ vs. $\left.A_{\mathrm{avg}}^{\mathrm{CPD}}=+0.06\right)$. The difference might originate from very sparse sampling of the top snow layers (Fig. 16, bottom right panel) because taking samples was difficult due to soft fresh snow. No samples could be taken from the top $4 \mathrm{~cm}$.

For CT-4, we can exclude limited penetration to cause the difference, despite occurring warm temperatures a few days before, because the copolar coherence (Fig. 9) and the temporal coherence (Leinss et al., 2015, Fig. 19) did not show any anomaly. However, we can not exclude the fact that the model assumption of oriented spheroids is a too strong assumption for the very dendritic shape of fresh fluffy snow.

The vertical structure of the anisotropy profiles agrees to our expectation regarding the meteorological conditions described in the caption of Fig. 16. The anisotropy profiles show vertical structures in older snow layers, as expected for the geometry of metamorphic snow which was exposed to temperature gradients. In contrast to the old layers, the top layers show horizontally aligned structures as we expect it for fresh snow. The fact that fresh snow is related to horizontal structures and therefore to a positive CPD makes it possible to use the CPD for fresh snow detection.

\subsection{Correlation between fresh snow and a positive CPD}

Settling of new snow can cause an increasingly positive anisotropy at intermediate times due to the horizontal alignment of dendrite backbones (Löwe et al., 2011). According to our theory, increasing anisotropies cause an increase of the $\mathrm{CPD}$. Therefore, a change in CPD can be used to detect fresh snow as done in Leinss et al. (2014b) using satellite data.

As fresh snow settles within a few days after deposition, we expected the CPD not to increase simultaneously with accumulation of fresh snow but rather to increase with a time lag $\tau$ after snowfall. The parameter $\tau$ characterized the time shift between snowfall and the buildup of horizontal structures. A value $\tau \approx 0$ indicates that the CPD increases instantaneously with snowfall, whereas $\tau>0$ gives an approximation for the time delay after which the growth of vertical structures (driven by temperature gradient) exceeds the buildup of a horizontal structures due to settling. For too large temporal offsets $\tau$ between a change of snow depth (SD) and a CPD change, no correlation is expected as temperature gradient metamorphism dominates the evolution of the CPD.

In the following, we analyze the correlation between changes in snow depth $\triangle \mathrm{SD}$ and a change in CPD, $\triangle \mathrm{CPD}$. The correlation is defined as

$$
\begin{aligned}
R= & \operatorname{corr}\{\mathrm{CPD}(t+\tau)-\mathrm{CPD}(t+\tau-\Delta T), \\
& \mathrm{SD}(t)-\mathrm{SD}(t-\Delta T)\},
\end{aligned}
$$

where SD is the measured snow depth, $\tau$ is the temporal offset between both time series as explained above, $\Delta T$ is the sampling interval, and $R$ is the Pearson correlation coefficient. The sampling interval $\Delta T$ is the time difference between two measurements of SD and the CPD and corresponds, for example, to the repeat time of satellite acquisitions. $\Delta T$ needs to be large enough to give fresh snow some time for settling such that the CPD increases above the phase noise level. However, the sampling time should not be too large, as minor snowfall events might be missed, and also 

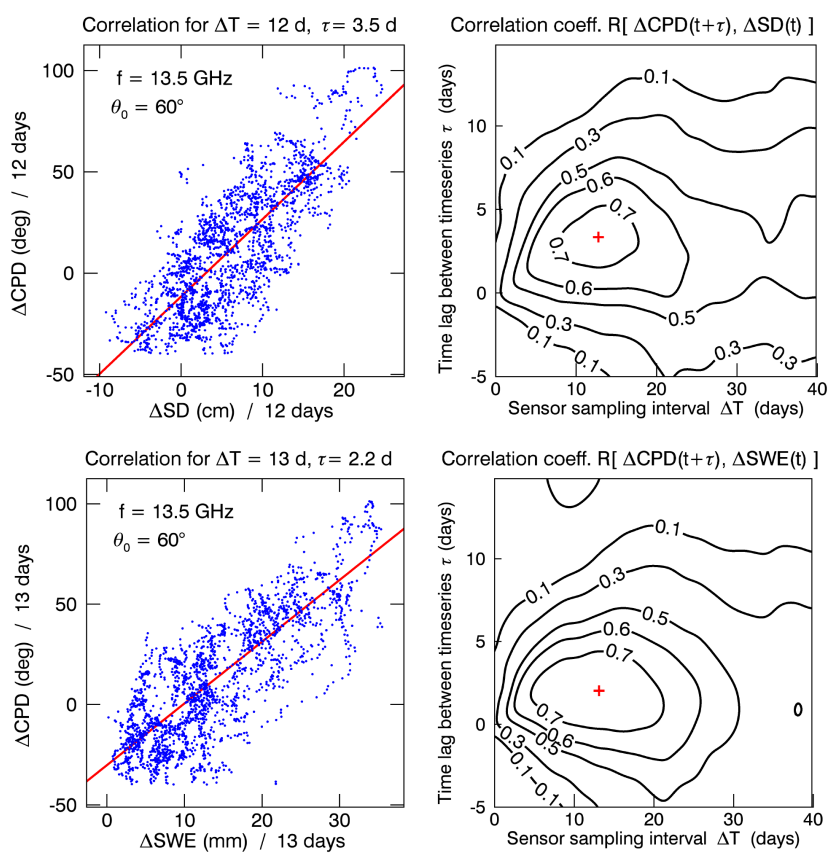

Figure 17. Left panels: correlation between $\triangle \mathrm{CPD}$ and changes in snow depth $\triangle \mathrm{SD}$ (top panel) and $\triangle \mathrm{SWE}$ (bottom panels) within a sampling interval of $\Delta T=12$ and 13 days. The time $t+\tau$ when the CPD difference was obtained is shifted by $\tau=3.5$ days (top panels) and $\tau=2.2$ days (bottom panels) vs. the time $t$ when the snow depth difference $\triangle \mathrm{SD}$ was obtained because the maximum CPD was always observed after fresh snow has already settled. Right panels: contour plots of the Pearson correlation coefficients $R$ for different pairs of $\Delta T$ and $\tau$. The red cross marks the pair $(\Delta T, \tau)$ with the highest correlation coefficient $R$.

snow metamorphosis will reduce measured values of positive CPD changes as they are typical for fresh snowfall.

The scatter plot in Fig. 17 (top panels) shows the correlation between the depth of fresh snow within 12 days and the corresponding change in CPD measured with a time lag of 3.5 days. The scatter plot is shown for the best correlation, $R=0.75$, which was found for different values of $\Delta T$ and $\tau$. The correlation coefficient $R$ is shown for all tested values of $\Delta t$ and $\tau$ in the contour plot of Fig. 17 (top right panel). The red cross marks the pair with the highest correlation coefficient.

The range of optimal sampling intervals, $\Delta T$, can be derived from the contour plot shown in Fig. 17. The plot shows an optimal $\Delta T$ of 9 to 15 days. For the correlation analysis, we evaluated all frequencies and incidence angles and the best correlation coefficients, ranging from 0.65 to 0.75 , were always found for $\Delta T=11 \pm 3$ days and a time lag of $\tau=3.0 \pm 0.5$ days.

The optimal sampling interval $\Delta T$ matches the 11-day orbit repeat time of TSX. Using time series of TSX, a CPD change of +10 to $+15^{\circ}$ per $10 \mathrm{~cm}$ of fresh snow was observed at $9.65 \mathrm{GHz}$ at an incidence angle of $33^{\circ}$ (Leinss et al., 2014b). From these results we would expect that the CPD changes by $40-60^{\circ}$ at the central frequency of the SSI of $13.5 \mathrm{GHz}$ at $\theta_{0}=60^{\circ}$. Here we observed a change in CPD of $38^{\circ}$ per $10 \mathrm{~cm}$ of fresh snow at $13.5 \mathrm{GHz}$, which fits well with respect to the uncertainty $R=0.74$ of Fig. 17 (top left panel).

The availability of accurate time series of the SWE measurements published in Leinss et al. (2015) made it also possible to check whether a correlation exists between $\triangle$ SWE and $\triangle \mathrm{CPD}$. The lower two graphs of Fig. 17 show an example for the correlation. The best correlations $(R \approx 0.65 \ldots 0.8)$ were found for a sampling interval of $\Delta T=10 \pm 3$ days with a time lag of $\tau=2.2 \pm 0.3$ days. The correlation with $\triangle \mathrm{SWE}$ is slightly better compared to the correlation with $\Delta \mathrm{SD}$.

\subsection{Comparison with satellite data}

The CPD observed by the ground-based SnowScat instrument could also be measured from space with the satellite TerraSAR-X. Spatial and temporal correlations between the CPD and snow depth were published by Leinss et al. (2014b). Figure 18 compares phase differences measured by TSX for the two seasons: 2011-2012 and 2012-2013. The space-borne measurements show the same trends as the ground-based measurements. However, the CPD values observed by TSX are about a factor of 2 smaller than the CPD measured with the SSI (scatter plot, Fig. 18). The reason is very likely that the TSX measurements were obtained from large open areas. In the large areas about $30 \%$ less snow depth was measured (Fig. 3 in Leinss et al., 2014b), probably due to a stronger wind exposition compared to the more wind-protected forest clearing where the SSI was located. Wind might also be a reason for disturbed snow settling as wind-drifted snow crystals show a different microstructure than undisturbed settled snow. The lower snow depth and the stronger wind exposition might explain why smaller phase differences were measured. Some residual vegetation and trees contained in the large areas observed by TSX also decreased the measured CPD due to spatial averaging.

\subsection{Effect of underlying soil}

Sector 2, as shown in Fig. 5, was covered with an metallic mesh by August 2011 to isolate purely snow-specific radar signatures from effects of the underlying soil. In the winter 2011/2012 strong ice built up on the mesh, causing high backscattering. However, we did not observe any effect on the CPD and the data of both sectors agree very well (Fig. 19, middle panel). To prevent the buildup of an ice crust in the next season, the mesh was cleared from ice on 12 December 2012 (vertical dashed line). The removal of the ice crust in the season 2012/2013 again did not affect the measured CPD much, and no large differences between the soil sector and the mesh sector were found. We could speculate that 


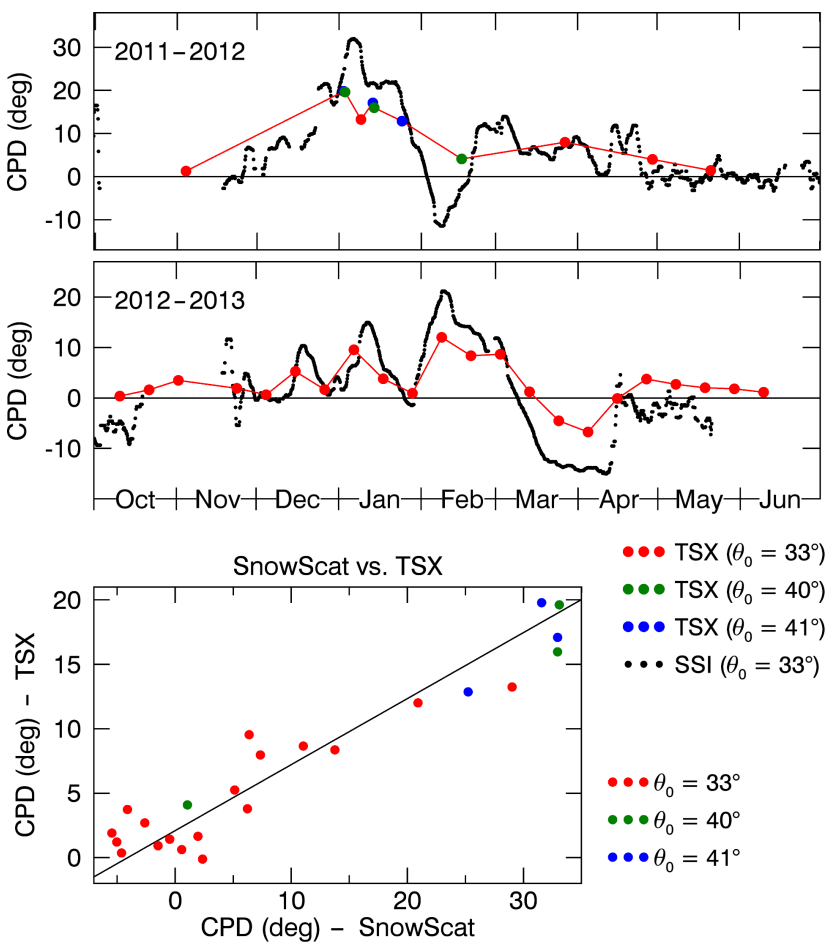

Figure 18. CPD measured by TerraSAR-X (TSX) at $\theta=33,40$, and $41^{\circ}$ compared to SnowScat (SSI) measurements ( $f=9.65 \mathrm{GHz}$, interpolated to $\theta=33^{\circ}$ ). Both instruments show the same trend (top panel) but the CPD measurements of the SSI are about a factor of two larger than the TSX measurements (bottom panel). The discrepancy can be explained by different snow conditions as the TSX data were acquired over large open areas where about $30 \%$ less snow depth was measured compared to the test site of the SSI.

slightly larger CPD values measured between January and April 2013 might indicate the missing of a layer of vertical oriented depth hoar crystals, but the deviation could also originate from slightly different snow conditions of the two sectors. Still, the good agreement between the measurements of the soil sector and the measurements from the metallic mesh confirms again that the measured CPD is almost purely a signal resulting from the snow volume. Although the CPD signal is caused by the snow volume, temperature gradient metamorphism alters the anisotropy of snow. As the temperature gradient is partially determined by the temperature of the underlying soil there exists an indirect effect of the soil energy balance to the evolution of the CPD.

\section{Conclusions}

We demonstrated a contact-less technique for monitoring the temporal evolution of the depth-averaged anisotropy of a seasonal snowpack. The technique is based on measuring the birefringent dielectric properties of snow at microwave frequencies where scattering effects can be neglected. The di-

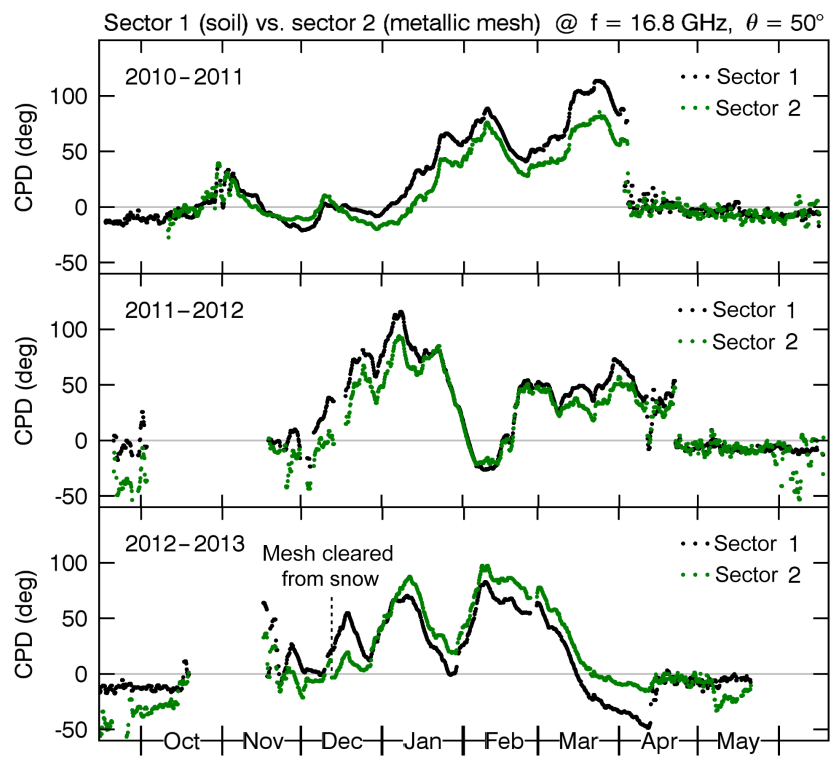

Figure 19. Comparison of CPD measurements from the two sectors of the test site. Sector 2 (green) was located between trees behind the SSI and was covered with a metallic mesh during the last two seasons of the experiment (after August 2011). Generally, the CPD of sector 2 evolves very similar to sector 1 and shows no large deviations.

electric and the structural anisotropy was determined from the CPD measured by a ground-based radar instrument complemented by the additionally required data for snow depth and density.

A theoretical framework was developed which describes the structural anisotropy of snow by oblate or prolate spheroidal ice grains and air inclusions, both of which have their symmetry axis in the vertical. Using MG-type mixing formulas, the effective permittivity tensor was calculated to describe the microwave birefringence of snow. To make contact to the microstructure characterization in previous work, we showed that the model of spheroidal inclusions is equivalent to a more general approach for the effective permittivity tensor based on correlation functions. From the effective permittivity tensor we calculated the birefringence and wave propagation according to anisotropic optics. The propagation delay difference of orthogonally polarized microwaves was described in terms of the CPD. The CPD depends linearly on frequency and anisotropy but shows only a weak dependence on density for the density range of seasonal snow. The CPD was then analyzed together with measured snow depth and density to estimate the dielectric and structural anisotropy averaged over snow depth.

Four years of polarimetric radar data from 10 to $17 \mathrm{GHz}$ were analyzed. The data were acquired by the SSI installed at a test site near the town of Sodankylä in Northern Finland. CPDs ranging from -30 to $+135^{\circ}$ were measured for $50-60 \mathrm{~cm}$ deep snow at a frequency of $13.5 \mathrm{GHz}$. The large 
variations of the CPD show that the anisotropy of snow is to be considered in polarimetric microwave studies of snowcovered regions.

Overall, depth-averaged dielectric anisotropies, $\varepsilon_{x}-\varepsilon_{z}$, were obtained ranging from -0.01 to +0.02 ; depth-averaged structural anisotropies $A$ between -0.05 and +0.25 were determined with a standard deviation of 0.005 obtained from measurements at different incidence angles and frequencies. Additional uncertainties originating from snow depth and density measurements were not taken into account, however.

To analyze deviations between modeled and measured CPD data, the CPD obtained from the electromagnetic model was calculated for different frequencies $(10 \ldots 17 \mathrm{GHz})$ and incidence angles $\left(30 \ldots 60^{\circ}\right)$ for the mean anisotropy $A_{\mathrm{avg}}^{\mathrm{CPD}}(t)$ obtained for each time $t$. The modeled CPD deviated by only $5-10^{\circ}$ from measured values ranging from -30 to $+135^{\circ}$. The expected linear frequency dependence of the CPD could be confirmed; the linear dependence also confirms that the CPD is a volumetric snow property which is determined by the dielectric anisotropy and is related to the structural anisotropy of the ice matrix and pore spaces of snow.

For four dates, the CPD-based anisotropy estimates were validated by micro-computed tomography $(\mu \mathrm{CT})$ measurements for which the anisotropy was computed directly from two-point correlation functions. In two cases, the $\mu \mathrm{CT}$ based depth-averaged anisotropy agreed with their CPDbased counterparts within 4 and $8 \%$. In one case we found a fair agreement, while for the fourth sample we found a larger deviation. The origin could only be hypothesized to result from missing snow samples, limitations of the MG mixing formulas, or limitations of using exponential correlation lengths to evaluate the anisotropy parameter $Q$.

In addition, we investigated the potential of CPD time series to detect the accumulation of fresh snow and the increase of SWE. A weak correlation was found and an optimal acquisition interval of 8-15 days was determined to detect the depth of fresh snow from CPD measurements. We found that the evolution of the CPD shows a delay of about 2-3 days compared to the evolution of snow depth, which indicates an average settling time of a few days.

The CPD measurements obtained from the ground-based instrument SnowScat were compared with space-borne data from the radar satellite TSX analyzed over large open areas located a few hundred meters from SnowScat. Both sensors showed the same temporal trend. However, the CPD observed by TSX was about a factor of 2 smaller than the SnowScat measurements. The reason could be spatial variability of snow depth and snow properties due to wind exposition but also some disturbing vegetation cover in the areas observed by TSX.

Our study shows that remote sensing techniques allow determination of the dielectric anisotropy of the snowpack when additional information about snow depth and a rough approximation of snow density are available. Currently, snow depth is mainly estimated from optical measurements such as photogrammetry (Marti et al., 2016; Bühler et al., 2015) or lidar instruments (Deems et al., 2013); however, the applicability of high-frequency radar instruments is currently discussed (Evans and Kruse, 2014). Snow density could potentially be derived from measurements of the snow water equivalent (Leinss et al., 2015) if data about the snow depth are available.

The possibility to observe the dielectric anisotropy of the snowpack by remote sensing techniques opens a new field of applications. Determination of the structural anisotropy and detection of fresh snow is discussed in this paper. In principle, the CPD measured over glaciers and ice sheets should provide some information about the structure of firn. However, the interpretation is difficult, though the depth of the scattering center for firn can be determined by independent means (Weber Hoen and Zebker, 2000).

Another interesting application is using CPD measurements as an indicator for the thermal conductivity of the snowpack. As the dielectric anisotropy can be exactly related to the anisotropy employed for parameterization of the thermal conductivity (Löwe et al., 2013) it seems feasible to aim at a proxy for the thermal conductivity from radar measurements, given a reasonable assumption about the mean density and snow depth. Thereby, the anisotropy would reflect predominant variations in the metamorphic state of the snowpack since increasing vertical structures are indicative of depth hoar. This might be important for the ground thermal regime in permafrost regions, where large vertical structures are created by high temperature gradients in the shallow snowpack in early winter. Depth hoar, with its large crystals and low density close to vegetation and soil in turn, is not only important for the survival of many rodents (Bilodeau et al., 2013) but is also very important for understanding the radar backscatter signal of snow (King and Derksen, 2015).

The large observation time spanning four winter seasons with a sampling interval of $4 \mathrm{~h}$ builds a unique dataset to study the evolution of snow anisotropy in order to gain further insight into the growth mechanisms of anisotropic snow crystals. Understanding the structural anisotropy of snow enhances the understanding of macroscopic anisotropic properties such as thermal conductivity, mechanical stability, and electromagnetic properties, especially the dielectric anisotropy. The developed method to measure snow anisotropy, its good agreement with ground-based $\mu \mathrm{CT}$ measurements, and the fair agreement with satellite-based radar measurement provide a unique opportunity to improve snow models and globally sense the metamorphic state of the snowpack. 


\section{Appendix A: Fabric anisotropy from crystal orientation}

For radio- and microwaves, single hexagonal ice crystals are dielectrically anisotropic, since the dielectric permittivity parallel to the $c$ axis, $\varepsilon_{\|}$, is by $\Delta \varepsilon_{\text {ice }}=\varepsilon_{\|}-\varepsilon_{\perp}=0.03 \ldots 0.04$ larger compared to the perpendicular permittivity $\varepsilon_{\perp}$ (Fujita et al., 1993; Matsuoka et al., 1997). Commonly, ice and snow occur as polycrystals which can be characterized by their $\mathrm{COF}$, i.e., the orientation of the $c$ axes. The COF can be described in terms of the orientation tensor $\mathbf{a}^{(2)}$. This second-order tensor is explained in Durand et al. (2006), nicely visualized by Woodcock (1977), and used in Riche et al. (2013) to characterize the crystals orientation in seasonal snow. The eigenvalues of $\mathbf{a}^{(2)}$ follow the relation $1 \geq \lambda_{1} \geq \lambda_{2} \geq \lambda_{3} \geq 0$ with $\lambda_{1}+\lambda_{2}+\lambda_{3}=1$ (Durand et al., 2006) and give the axis of an ellipsoid.

For snow with a vertical symmetry axis $(z)$ the eigenvalues correspond to the axes of an ellipsoid aligned along the coordinate axis $i=\{x, y, z\}$. The ellipsoid can have the following three shapes: (1) for a preferential horizontal orientation of the $c$ axis (horizontal girdle) the eigenvalues $\left(0 \leq \lambda_{z}<1 / 3<\lambda_{y} \approx \lambda_{z} \leq 1 / 2\right)$ form an oblate spheroid; (2) for an isotropic distribution, the eigenvalues form a sphere $\left(\lambda_{x}=\lambda_{y}=\lambda_{z}=1 / 3\right)$; (3) when the $c$-axis clusters around the vertical (single maximum fabric), the eigenvalues form a prolate spheroid $\left(0 \leq \lambda_{x} \approx \lambda_{y}<1 / 3<\lambda_{z} \leq 1\right)$.

The effective permittivity of snow, $\varepsilon_{\text {eff }}$, composed of air and a matrix of (isotropically) oriented ice crystals can be described by mixing formulas (e.g., MG as in Sect. 2.2). In the following, we parameterize the effective permittivities $\varepsilon_{i \text {,eff-oi }}$ of snow comprising oriented ice crystals by a weighted average of the two permittivities of ice, $\varepsilon_{\|}$and $\varepsilon_{\perp}$. The weighting is determined by the eigenvalues of $\mathbf{a}^{(2)}$. The weight for $\varepsilon_{\|}$is equivalent to $\lambda_{i}$; the weight for $\varepsilon_{\perp}$ in the perpendicular direction follows $\left(1-\lambda_{i}\right)$. The effective permittivities of snow for the $x, y$, and $z$ direction follow as

$\varepsilon_{i, \text { eff-oi }}=f(\rho) \cdot\left[\lambda_{i} \cdot \varepsilon_{\|}+\left(1-\lambda_{i}\right) \cdot \varepsilon_{\perp}\right]$.

The function $f(\rho)=\varepsilon_{\text {eff }}\left(f_{\text {vol }}, \varepsilon_{\text {air }}, \varepsilon_{\text {ice }}\right) / \varepsilon_{\text {ice }}$ accounts for the nonlinear density dependence of the permittivity of snow (Eq. 4) parameterized by the ice volume fraction $f_{\mathrm{vol}}$ and by the dielectric constant of polycrystalline of ice, $\varepsilon_{\text {ice }}$.

The dielectric anisotropy of snow resulting from oriented ice crystals, $\Delta \varepsilon_{\text {snow-oi }}=\varepsilon_{x \text {,eff-oi }}-\varepsilon_{z \text {,eff-oi }}$, can now be related to the dielectric anisotropy of ice $\Delta \varepsilon_{\text {ice }}$ by

$\Delta \varepsilon_{\text {snow-oi }}=\varepsilon_{x, \text { eff-oi }}-\varepsilon_{z, \text { eff-oi }}=f(\rho) \cdot\left(\lambda_{x}-\lambda_{z}\right) \Delta \varepsilon_{\text {ice }}$.

For the two extreme cases of snow with completely vertically oriented ice crystals $\left(\lambda_{x, y}=0, \lambda_{z}=1\right)$, it follows that $\Delta \varepsilon_{\text {snow-oi }}=-f(\rho) \Delta \varepsilon_{\text {ice }}$; for snow with a uniform orientation in the horizontal plane $\left(\lambda_{x, y}=0.5, \lambda_{z}=0\right)$ one obtains $\Delta \varepsilon_{\text {snow-oi }}=+\frac{1}{2} f(\rho) \Delta \varepsilon_{\text {ice }}$. For the isotropic case $\left(\lambda_{i}=1 / 3\right)$ one obtains $\Delta \varepsilon_{\text {snow-oi }}=0$. The square bracketed term in Eq. (A1) is then equivalent to the permittivity of polycrystalline ice, $\varepsilon_{\text {ice }}=1 / 3 \cdot \varepsilon_{\|}+2 / 3 \cdot \varepsilon_{\perp}$. This one-third/two-third weighting for polycrystalline ice has also been mentioned by Fujita et al. (1993, 2000) and was experimentally observed by Matsuoka et al. (1996).

For seasonal snow, some evidence has been found that the $c$ axis is preferentially vertically aligned for fresh snow (single-maximum), whereas for old snow the $c$ axis seems to be slightly oriented in a horizontal girdle (Riche et al., 2013). The strongest (single-maximum) anisotropy observed by Riche et al. (2013), parameterized by the eigenvalues $\lambda_{z}=0.53, \lambda_{x}=0.22$, results with $\mathrm{Eq}$. (A2) in a maximum dielectric anisotropy of $\Delta \varepsilon_{\text {snow }} \approx f(\rho) \cdot(0.22$ $0.53) \cdot 0.035=-0.002$ with $f(\rho) \approx f_{\mathrm{vol}}=0.2$ as common for seasonal snow. According to Fig. 1(right panel) this would correspond to a structural anisotropy of $A=-0.02$, which is small compared to the structural anisotropies (Fig. 11) observed in this paper.

\section{Appendix B: Effective permittivity from weighted average of Maxwell-Garnett equations}

The MG formulas (e.g., Eq. (3.27) in Sihvola, 2000) describe the effective permittivity of (elliptical) inclusion with a permittivity $\varepsilon_{\mathrm{i}}$ (e.g., ice) embedded in a host medium of permittivity $\varepsilon_{\mathrm{e}}$ (e.g., air) and can therefore be applied to calculate the anisotropic permittivity tensor for media with a structural anisotropy. The MG formulas can also be applied in an "inverted" form, where the permittivities of inclusions and the host medium are swapped (e.g., air inclusions in ice). Both the MG formula and the "inverse" MG formula, Eqs. (6a) and (6b), are equivalent to the lower and upper HSBs for approximations of the exact description of dielectric mixtures (Hashin and Shtrikman, 1962; Sihvola, 2002).

For the isotropic case, we compared the MG formulas to measurements of Mätzler (1996) and found that the measurements lie well within the lower and upper HSBs (Sihvola, 2002). However, the measurements are significantly larger than the lower HSB (MG for ice inclusion in air). Therefore, a combination of MG and its inverse was constructed for better agreement of the modeled and measured permittivity.

The measured data of Mätzler (1996) have been used in MEMLS-3 (Wiesmann and Mätzler, 1999, Eqs. 45/46) to fit an empirical formula for the permittivity of dry snow. The empirical formula is given in terms of snow density $\rho$ by

$\varepsilon_{\mathrm{MEM}-3}=\left\{\begin{array}{ll}1+1.5995 \rho+1.861 \rho^{3} & \rho<0.4 \mathrm{~g} \mathrm{~cm}^{-3} \\ {\left[(1-v) \varepsilon_{h}+v \varepsilon_{\text {ice }}^{1 / 3}\right]^{3}} & \rho>0.4 \mathrm{~g} \mathrm{~cm}^{-3}\end{array}\right.$,

with the coefficients $\varepsilon_{h}=1.005, \varepsilon_{\text {ice }}=3.17$ (for $-10^{\circ} \mathrm{C}$ ), and $v=\rho / \rho_{\text {ice. Note }}$ that the original coefficients of $\varepsilon_{h}=1.0, \varepsilon_{\text {ice }}=3.215$ given by Wiesmann and Mätzler (1999, Eqs. 45/46) have been adapted in agreement with C. Mätzler to produce correct results for pure ice $(v=1)$. We also note here that the exponent $1 / 3$ is missing for the factor $\varepsilon_{\mathrm{S}}$ (here $\left.\varepsilon_{\text {ice }}\right)$ in Wiesmann and Mätzler (1999, Eq. 46). 

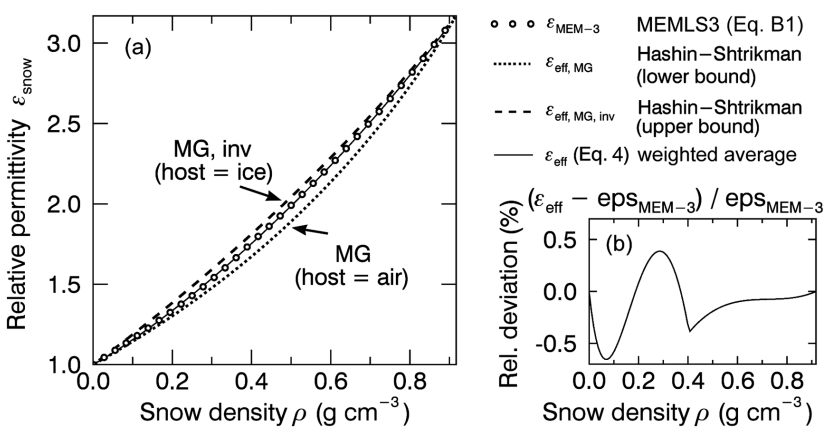

Figure B1. (a) Relative permittivity of dry snow $\varepsilon_{\text {MEM-3 }}$ (open circles) according to Eq. (B1) in comparison to the upper and lower Hashin-Shtrikman bound. The solid lines shows the weighted average of the MG formulas, $\varepsilon_{\text {eff }}$, Eq. (4). (b) The relative deviation of $\varepsilon_{\text {eff }}$ with respect to $\varepsilon$ MEM-3 is below $0.7 \%$ for all snow densities.

Figure B1a shows the lower and upper HSBs (MG and MG,inv, dashed and dotted line) and the result of Eq. (B1) (solid line). The solid dots indicate the weighted average of both bounds as given in Eq. (4). The weighted average is motivated by the fact that for low densities snow can better be described by ice particles embedded in a background matrix of air, whereas for high densities snow can better be described by air inclusions in ice. Figure B1b shows the relative deviation (in percent) between the permittivity used in MEMLS-3 (Eq. B1) and the weighted average of the MG formula and its "inverse" form. The relative deviation $\left(\varepsilon_{\text {eff }}-\varepsilon_{\text {MEM-3 }}\right) / \varepsilon_{\text {MEM-3 }}$ is less than $\pm 0.7 \%$ and justifies the phenomenological composition of the averaging formula.

\section{Appendix C: Re-derivation of Maxwell-Garnett equations via correlation functions}

In Rechtsman and Torquato (2008) an exact series expansion of the dielectric permittivity of arbitrary anisotropic twophase materials was derived and related to the $n$ point correlation functions of the material. When the series is truncated at $n=2$, the final result (Rechtsman and Torquato, 2008, Eq. 16) can be solved for the diagonal components, $\varepsilon_{\mathrm{eff}, i}$, $i=x, y, z$, of the effective permittivity tensor which can be written in the form

$\varepsilon_{\mathrm{eff}, i}=\varepsilon_{q}+\varepsilon_{q} \phi_{p} \frac{\left(\varepsilon_{p}-\varepsilon_{q}\right)}{\varepsilon_{q}+\left(1-\phi_{p}\right)\left[\frac{1}{3}-\frac{U_{i}}{3 \phi_{p} \phi_{q}}\right]\left(\varepsilon_{p}-\varepsilon_{q}\right)}$.

The permittivities and volume fractions of the two phases composing the microstructure are denoted by $\varepsilon_{p}, \varepsilon_{q}$ and $\phi_{p}, \phi_{q}$, respectively. The quantities $U_{i}$ in Eq. (C1) are related to integrals over the two-point correlation function $C(\boldsymbol{r})$ defined in Löwe et al. (2013, Eq. 1). In the lowest order of frequency $f$, contributions to the effective permittivity from scattering can be neglected (cf. Rechtsman and Torquato, 2008, Eqs. C3, C4). Then the $U_{i}$ have vanishing imaginary parts and are given by

$$
\begin{aligned}
& U_{x}=U_{y}=\frac{3}{4 \pi} \int_{\mathbb{R}^{3}} d^{3} r \frac{1}{r^{3}}\left(-1+\frac{3}{2} \sin ^{2} \theta\right) C(\boldsymbol{r}), \\
& U_{z}=\frac{3}{4 \pi} \int_{\mathbb{R}^{3}} d^{3} r \frac{1}{r^{3}}\left(-1+3 \cos ^{2} \theta\right) C(\boldsymbol{r}) .
\end{aligned}
$$

Here $r=|\boldsymbol{r}|$ is the magnitude of $\boldsymbol{r}$ and $\theta$ denotes the angle between the vertical $z$ axis and $\boldsymbol{r}$.

When the microstructure is (statistically) transversely isotropic, it is reasonable to assume a "spheroidal symmetry" of the correlation function, viz $C(\boldsymbol{r})=C(r / \sigma(\theta))$ with $\sigma(\theta)=2 a_{x}\left[1-\left(1-a_{x}^{2} / a_{z}^{2}\right) \cos ^{2} \theta\right]^{1 / 2}$ as used in Löwe et al. (2013). Under this assumption, the singular integrals in Eq. (C2) can be calculated as shown in Torquato and Lado (1991). The results can be inserted into the square brackets in Eq. (C1), yielding

$\left[\frac{1}{3}-\frac{U_{x}}{3 \phi_{p} \phi_{q}}\right]=Q$ and $\left[\frac{1}{3}-\frac{U_{z}}{3 \phi_{p} \phi_{q}}\right]=1-2 Q$,

where the anisotropy parameter $Q$ is defined in Löwe et al. (2013, Eq. 4) or Torquato (2002, Eqs. 17.30/17.31). Using the definition of depolarization factors from Torquato (2002, Eq. 17.25), noting their relation to $Q$ from Torquato (2002, Eq. 17.29) on one hand and their equivalence to the definition of $N_{\mathrm{i}}$ by Eq. (8) on the other hand, we end up with

$\varepsilon_{\mathrm{eff}, i}=\varepsilon_{q}+\varepsilon_{q} \phi_{p} \frac{\left(\varepsilon_{p}-\varepsilon_{q}\right)}{\varepsilon_{q}+\left(1-\phi_{p}\right) N_{\mathrm{i}}\left(\varepsilon_{p}-\varepsilon_{q}\right)}$.

We note here that Torquato (2002, Eq. 17.25) contains a typo. Specifying, $p$ as the ice phase and $q$ as the air phase in Eq. (C5) gives $\varepsilon_{q}=\varepsilon_{\text {air }}, \varepsilon_{p}=\varepsilon_{\text {ice }}, \phi_{p}=f_{\text {vol }}$ in the notation from Sect. 2.2, and thus Eq. (C5) coincides with the MG result Eq. (6a).

\section{Appendix D: CPD calibration of the SnowScat data}

The measured radar signal was calibrated by an internal calibration loop of the SSI to compensate system drifts. However, some polarization-dependent signal delay still originated from the connectors of the antenna feeding cables and from the antennas themselves due to the polarizationdependent beam pattern. In order to calibrate external offsets and drifts, the CPD was calibrated with two metallic targets.

The primary calibration target was a metallic sphere with a diameter of $25 \mathrm{~cm}$ mounted on a wooden pole during the experiment. The sphere can be located in Fig. 5 next to the SSI. A secondary target, a metallic plate, was located behind trees close to sector 2. A third calibration target, a dihedral reflector, was installed during the setup phase of the experiment. The correct antenna pointing direction to the sphere was determined with a precision of $\pm 0.5^{\circ}$ by 2 -D scans in 
elevation and azimuth. The 2-D scans showed that possible systematic error of the CPD caused by imprecise alignment are smaller than $\pm 10^{\circ}$.

The theoretical CPD measured from a sphere (or plate) is 0 due to the target symmetry. The sphere was measured every $4 \mathrm{~h}$ and was used as a reference during the whole duration of the experiment. The plate was installed from October 2011 until June 2013 and was used to validate the calibration done with the sphere. The CPD measured for a dihedral reflector should be $180^{\circ}$. The dihedral reflector was measured once, on 9 December 2009, to verify the processing sequence of the SnowScat raw data.

The CPD determined for the sphere, $\mathrm{CPD}_{\mathrm{REF}}$, was used as a reference and was subtracted from the uncalibrated CPD measurements, $\mathrm{CPD}_{\text {uncal., }}$, to obtain calibrated results:

$\operatorname{CPD}_{\text {cal. }}(f)=\operatorname{CPD}_{\text {uncal. }}(f)-\operatorname{CPD}_{\mathrm{REF}}(f)$.

Phase unwrapping was performed for the uncalibrated CPD and the reference CPD when necessary.

To reduce noise in the reference measurements as much as possible, the reference, $\mathrm{CPD}_{\mathrm{REF}}$, was determined as follows: time series $\operatorname{CPD}_{\mathrm{REF}}(t)$ were obtained for 21 different frequencies in order to sample the entire frequency spectrum between 9.2 and $17.8 \mathrm{GHz}$ of the instrument. The time series were smoothed with a median filter of 4 days, which preserved phase jumps in the signal. After temporal filtering, a frequency-dependent fourth-order polynomial was fitted over the measured frequency spectrum of each acquisition to provide some noise reduction in the frequency domain. The (frequency-dependent) reference $\operatorname{CPD}_{\mathrm{REF}}(f)$ is shown for $f=13.5 \mathrm{GHz}$ in Fig. D1 (solid black line). Individual measurements of the sphere as well as measurements of the metallic plate are shown as dark and light gray solid dots below the black line.

In the third season, between 18 November 2011 and 20 January 2012, the pointing direction (elevation angle) to the sphere was misaligned by $2^{\circ}$. Therefore, the reference $\mathrm{CPD}$ was corrected by a frequency dependent offset to keep the CPD continuous at the start and end of the misalignment period.

The deviation of the raw data of the sphere from the reference, $\triangle \mathrm{CPD}=\mathrm{CPD}(f)-\mathrm{CPD}_{\mathrm{REF}}(f)$, is shown in the lower panels for each season as scattered dots for each of the 21 analyzed frequencies. The root-mean-square error was below $4^{\circ}$ for the full frequency spectrum and is given for each seasons next to the graph. The error of the reference, $\operatorname{CPD}_{\operatorname{REF}}(f)$, which includes systematic and statistic errors, is estimated to be below $15^{\circ}$.
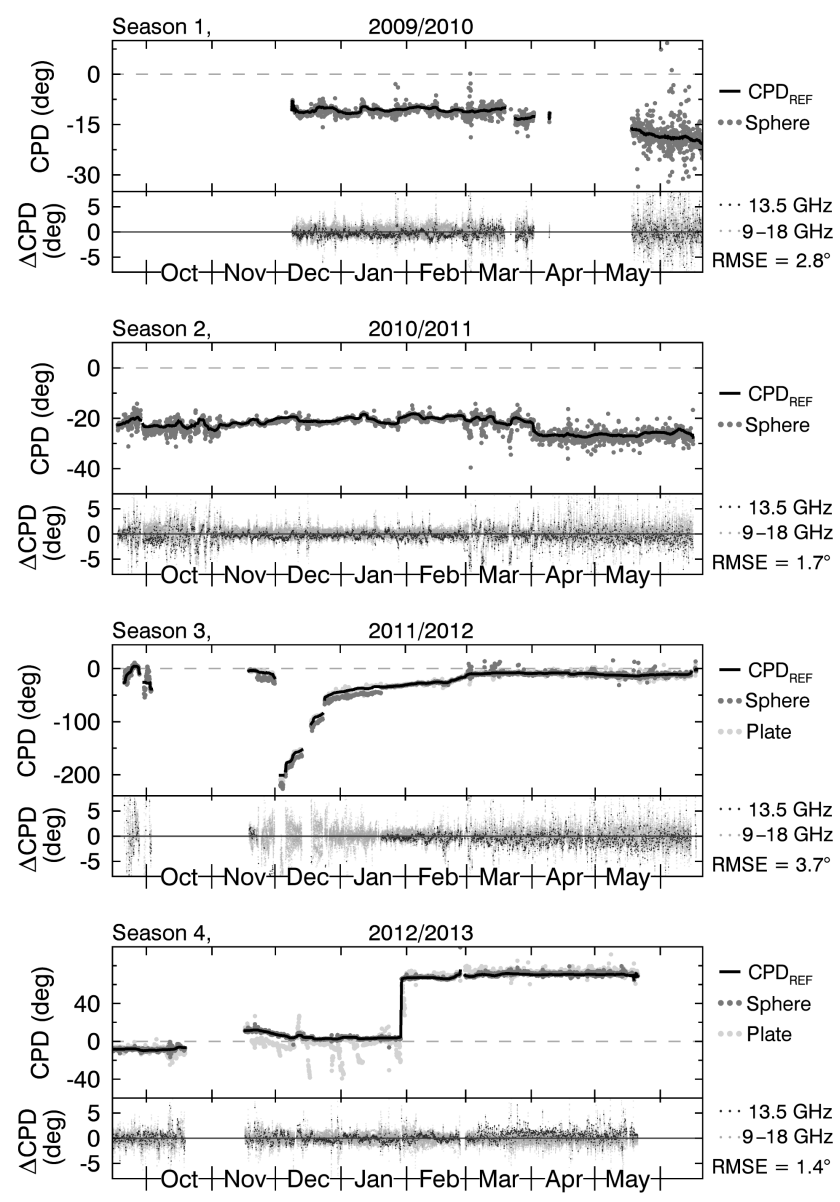

Figure D1. The CPD was calibrated using the sphere as a reference target. The upper panels show the reference, $\operatorname{CPD}_{\operatorname{REF}}(f)$, for $f=13.5 \mathrm{GHz}$ (solid line) together with individual CPD measurements for the sphere and the plate (light and dark gray bullets). The CPD of the metallic plate agrees within the standard deviation with measurements of the sphere and with CPD $\mathrm{REF}_{\text {. }}$ Deviations were found for season 3 due to misalignment of the SSI to the sphere and for November 2012 due possibly to snow cover of the metallic plate. The lower panels show the deviation, $\Delta \mathrm{CPD}=\mathrm{CPD}_{\text {meas. }}(f)-\mathrm{CPD}_{\mathrm{REF}}(f)$, for individual measurements at all measured frequencies $f=10 \ldots 17 \mathrm{GHz}$. The deviation for $f=13.5 \mathrm{GHz}$ is shown as black dots. The standard deviation (RMSE) of $\triangle \mathrm{CPD}$ for the whole frequency spectrum is given below the legend of the lower panel. 
Author contributions. Silvan Leinss developed the birefringence model of snow, processed and calibrated the radar data, and wrote major parts of the manuscript. Henning Löwe established the link between the MG theory and the statistical description of the snow microstructure based on $n$-point correlation functions (Appendix C) and did the statistical analysis of the $\mu \mathrm{CT}$ data. Martin Proksch acquired the snow samples and calculated the anisotropy from the $\mu \mathrm{CT}$ data. Juha Lemmetyinen provided the snow measurements and meteorological data for the test site and added valuable "in situ" knowledge. Andreas Wiesmann added valuable knowledge about the processing and datasets of the SnowScat instrument. Irena Hajnsek carefully proofread the paper and provided the funding.

Acknowledgements. The in situ data collection was supported by the European Space Agency activity "Technical assistance for the deployment of an X- to Ku-band scatterometer during the NoSREx experiment" (ESA ESTEC contract no. 22671/09/NL/JA/ef) (Lemmetyinen et al., 2013). We acknowledge Margret Matzl from SLF for retrieving an additional $\mu \mathrm{CT}$ dataset from the archive. The staff at FMI-ARC is acknowledged for the collection of in situ data. We thank Anna Kontu (FMI) for providing the meteorological data and her excellent local expertise concerning many experimental details at the test site. Special thank goes to Jouni Pulliainen from FMI for the initiative of setting up a test site which provides a unique amount and diversity of meteorological data and snow measurements. We thank Helmut Rott from University Innsbruck/Enveo for the encouragement to analyze the CPD of the ground-based radar instrument SnowScat. We thank Christian Mätzler for adapting his model of the relative permittivity of snow in order to make it valid for high snow densities up to solid ice and for providing insight into his excellent work. We also acknowledge the anonymous reviewer for the very precise feedbacks, the careful checking of references, and differencing the structural anisotropy of snow from the crystal orientation fabric anisotropy of ice crystals.

Edited by: G. Chambon

Reviewed by: two anonymous referees

\section{References}

Alley, R.: Texture of polar firn for remote sensing, Ann. Glaciol., 9, $1-4,1987$.

Ao, C. O. and Kong, J. A.: Analytical approximations in multiple scattering of electromagnetic waves by aligned dielectric spheroids, J. Opt. Soc. Am. A, 19, 1145-1156, 2002.

Bilodeau, F., Gauthier, G., and Berteaux, D.: The effect of snow cover on lemming population cycles in the Canadian High Arctic, Oecologia, 172, 1007-1016, doi:10.1007/s00442-012-25498, 2013.

Bohleber, P., Wagner, N., and Eisen, O.: Permittivity of ice at radio frequencies: Part II. Artificial and natural polycrystalline ice, Cold Reg. Sci. Technol., 83-84, 13-19, doi:10.1016/j.coldregions.2012.05.010, 2012.

Bormann, K. J., Westra, S., Evans, J. P., and McCabe, M. F.: Spatial and temporal variability in seasonal snow density, J. Hydrol., 484, 63-73, doi:10.1016/j.jhydrol.2013.01.032,2013.
Bühler, Y., Marty, M., Egli, L., Veitinger, J., Jonas, T., Thee, P., and Ginzler, C.: Snow depth mapping in high-alpine catchments using digital photogrammetry, The Cryosphere, 9, 229243, doi:10.5194/tc-9-229-2015, 2015.

Calonne, N., Geindreau, C., Flin, F., Morin, S., Lesaffre, B., Rolland du Roscoat, S., and Charrier, P.: 3-D image-based numerical computations of snow permeability: links to specific surface area, density, and microstructural anisotropy, The Cryosphere, 6 , 939-951, doi:10.5194/tc-6-939-2012, 2012.

Calonne, N., Flin, F., Geindreau, C., Lesaffre, B., and Rolland du Roscoat, S.: Study of a temperature gradient metamorphism of snow from 3-D images: time evolution of microstructures, physical properties and their associated anisotropy, The Cryosphere, 8, 2255-2274, doi:10.5194/tc-8-2255-2014, 2014.

Chang, P., Mead, J., Knapp, E., Sadowy, G., Davis, R., and McIntosh, R.: Polarimetric backscatter from fresh and metamorphic snowcover at millimeter wavelengths, IEEE T. Anten. Propag., 44, 58-73, doi:10.1109/8.477529, 1996.

Cloude, S.: Polarisation - applications in remote sensing, Oxford University Press, New York, 2010.

Cohn, E.: Das elektromagnetische Feld: Vorlesungen über die Maxwell'sche Theorie, S. Hirzel, Leipzig, 43-57, 1900.

Davis, R. E. and Dozier, J.: Stereological characterization of dry Alpine snow for microwave remote sensing, Adv. Space Res. 9, 245-251, doi:10.1016/0273-1177(89)90492-4, 1989.

Deems, J. S., Painter, T. H., and Finnegan, D. C.: Lidar measurement of snow depth: a review, J. Glaciol., 59, 467-479, 2013.

Durand, G., Gagliardini, O., Thorsteinsson, T., Svensson, A., Kipfstuhl, S., and Dahl-Jensen, D.: Ice microstructure and fabric: an up-to-date approach for measuring textures, J. Glaciol., 52, 619630, doi:10.3189/172756506781828377, 2006.

Evans, J. and Kruse, F.: Determination of snow depth using elevation differences determined by interferometric SAR (InSAR), in: 2014 IEEE International Geoscience and Remote Sensing Symposium (IGARSS), 13-18 July 2014, Quebec, 962-965, doi:10.1109/IGARSS.2014.6946586, 2014.

Evans, S.: Dielectric properties of ice and snow - A review, J. Glaciol., 5, 773-792, 1965.

Fujita, S., Mae, S., and Matsuoka, T.: Dielectric anisotropy in ice Ih at $9.7 \mathrm{GHz}$, Ann. Glaciol., 17, 276-276, 1993.

Fujita, S., Matsuoka, T., Ishida, T., Matsuoka, K., and Mae, S.: A summary of the complex dielectric permittivity of ice in the megahertz range and its application for radar sounding of polar ice sheets, in: The Physics of Ice Core Records, First edition, Hokkaido University Press, Sapporo, Japan, 185-212, 2000.

Fujita, S., Maeno, H., and Matsuoka, K.: Radio-wave depolarization and scattering within ice sheets: a matrix-based model to link radar and ice-core measurements and its application, Journal of Glaciology, 52, 407-424, 2006.

Fujita, S., Okuyama, J., Hori, A., and Hondoh, T.: Metamorphism of stratified firn at Dome Fuji, Antarctica: A mechanism for local insolation modulation of gas transport conditions during bubble close off, J. Geophys. Res.-Ea. Surf., 114, 1-21, doi:10.1029/2008JF001143, 2009.

Fujita, S., Hirabayashi, M., Goto-Azuma, K., Dallmayr, R., Satow, K., Zheng, J., and Dahl-Jensen, D.: Densification of layered firn of the ice sheet at NEEM, Greenland, J. Glaciol., 60, 905-921, doi:10.3189/2014JoG14J006, 2014. 
Fujita, S., Goto-Azuma, K., Hirabayashi, M., Hori, A., Iizuka, Y., Motizuki, Y., Motoyama, H., and Takahashi, K.: Densification of layered firn in the ice sheet at Dome Fuji, Antarctica, J. Glaciol., 62, 103-123, doi:10.1017/jog.2016.16, 2016.

Garrett, T. J., Fallgatter, C., Shkurko, K., and Howlett, D.: Fall speed measurement and high-resolution multi-angle photography of hydrometeors in free fall, Atmos. Meas. Tech., 5, 26252633, doi:10.5194/amt-5-2625-2012, 2012.

Guneriussen, T., Høgda, K. A., Johnsen, H., and Lauknes, I.: InSAR for estimation of changes in snow water equivalent of dry snow, IEEE T. Geosci. Remote, 39, 2101-2108, doi:10.1109/36.957273, 2001.

Hallikainen, M., Ulaby, F., and van Deventer, T.: Extinction Behavior of Dry Snow in the 18-to 90-GHz Range, IEEE T. Geosci. Remote, GE-25, 737-745, doi:10.1109/TGRS.1987.289743, 1987.

Hargreaves, N.: The polarization of radio signals in the radio echo sounding of ice sheets, J. Phys. D, 10, 1285, doi:10.1088/00223727/10/9/012, 1977.

Hargreaves, N.: The radio-frequency birefringence of polar ice, J. Glaciol., 21, 301-313, 1978.

Hashin, Z. and Shtrikman, S.: A Variational Approach to the Theory of the Effective Magnetic Permeability of Multiphase Materials, J. Appl. Phys., 33, 3125-3131, doi:10.1063/1.1728579, 1962.

Heggli, M., Frei, E., and Schneebeli, M.: Instruments and Methods Snow replica method for three-dimensional Xray microtomographic imaging, J. Glaciol., 55, 631-639, doi:10.3189/002214309789470932, 2009.

Hendry, A., Mccormick, G., and Barge, B.: Ku-band and S-band observations of the differential propagation constant in snow, IEEE T. Anten. Propag., 24, 521-525, doi:10.1109/TAP.1976.1141364, 1976.

Hildebrand, T., Laib, A., Müller, R., Dequeker, J., and Rüegsegger, P.: Direct three-dimensional morphometric analysis of human cancellous bone: microstructural data from spine, femur, iliac crest, and calcaneus, J. Bone Miner Res., 14, 1167-1174, 1999.

Hogan, R. J., Tian, L., Brown, P. R. A., Westbrook, C. D., Heymsfield, A. J., and Eastment, J. D.: Radar scattering from ice aggregates using the horizontally aligned oblate spheroid approximation, J. Appl. Meteor. Clim., 51, 655-671, 2012.

Hörhold, M. W., Albert, M. R., and Freitag, J.: The impact of accumulation rate on anisotropy and air permeability of polar firn at a high-accumulation site, J. Glaciol., 55, 625-630, 2009.

Izumi, K. and Huzioka, T.: Studies of metamorphism and thermal conductivity of snow, 1, Low Temp. Sci. Ser. A, 33, 91-102, 1975.

Jones, R. G.: The measurement of dielectric anisotropy using a microwave open resonator, J. Phys. D, 9, 819-827, 1976.

King, J. and Derksen, C.: Retrieval of Tundra SWE Using Airborne Dual-frequency SAR (17.2/9.6 Ghz) and MEMLS-active, in: Proceedings IGARSS 2015, Milano, 2015.

Kojima, K.: Thin Section of Snow Cut by a Heated Wire, Contrib. Inst. Low Temp. Sci., 16, 47-59, 1960.

Kontu, A., Lemmetyinen, J., Takala, M., Rautiainen, K., Wiesmann, A., and Werner, C.: Technical assistance for the deployment of an X- to Ku-band scatterometer during the NoSREx experiment, Data acuisition report (D2), Contract No. 22671/09/NL/JA, Tech. rep., FMI, Enveo, Gamma Remote Sensing and ESA ESTEC, Noordwijk, 2011.
Landau, L. and Lifshitz, E.: Electrodynamics of Continuous Media, The MacMillian Company, New York, printed in Bristol, UK, 20-30, 1960.

Lee, J.-S. and Pottier, E.: Polarimetric Radar Imaging, CRC Press Taylor \& Francis Group, Boca Raton, 61-63, 2009.

Leinss, S., Lemmetyinen, J., Wiesmann, A., and Hajnsek, I.: Snow Structure Evolution Measured by Ground Based Polarimetric Phase Differences, in: EUSAR 2014, Proceedings of 10th European Conference on Synthetic Aperture Radar, 3-5 June 2014, Berlin, 1-4, 2014a.

Leinss, S., Parrella, G., and Hajnsek, I.: Snow Height Determination by Polarimetric Phase Differences in X-Band SAR Data, IEEE J. Select. Top. Appl. Earth Obs. Remote S., 7, 3794-3810, doi:10.1109/JSTARS.2014.2323199, 2014b.

Leinss, S., Wiesmann, A., Lemmetyinen, J., and Hajnsek, I.: Snow Water Equivalent of Dry Snow Measured by Differential Interferometry, IEEE J. Select. Top. Appl. Earth Obs. Remote S., 8, 3773-3790, doi:10.1109/JSTARS.2015.2432031, 2015.

Lemmetyinen, J., Kontu, A., Leppänen, L., Pulliainen, J., Wiesmann, A., Werner, C., Proksch, M., and Schneebeli, M.: Technical assistance for the deployment of an X- to Ku-band scatterometer during the NoSREx experiment, NoSREx-I, -II and III (2009-2012) Final report, Contract No. 22671/09/NL/JA/ef, Tech. rep., ESA ESTEC, Noordwijk, https://earth.esa.int/web/ guest/campaigns (last access: 26 July 2016), 2013.

Li, L., Gaiser, P., Albert, M., Long, D., and Twarog, E.: WindSat Passive Microwave Polarimetric Signatures of the Greenland Ice Sheet, IEEE T. Geosci. Remote, 46, 2622-2631, doi:10.1109/TGRS.2008.917727, 2008.

Lomonaco, R., Albert, M., and Baker, I.: Microstructural evolution of fine-grained layers through the firn column at Summit, Greenland, J. Glaciol., 57, 755-762, doi:10.3189/002214311797409730, 2011.

Löwe, H. and Picard, G.: Microwave scattering coefficient of snow in MEMLS and DMRT-ML revisited: the relevance of sticky hard spheres and tomography-based estimates of stickiness, The Cryosphere, 9, 2101-2117, doi:10.5194/tc-9-2101-2015, 2015.

Löwe, H., Spiegel, J., and Schneebeli, M.: Interfacial and structural relaxations of snow under isothermal conditions, J. Glaciol., 57, 499-510, 2011.

Löwe, H., Riche, F., and Schneebeli, M.: A general treatment of snow microstructure exemplified by an improved relation for thermal conductivity, The Cryosphere, 7, 1473-1480, doi:10.5194/tc-7-1473-2013, 2013.

Lüneburg, E. and Boerner, W.-M.: Statistical Aspects of Radar Polarimetry, in: Fields, Networks, Computational Methods, and Systems in Modern Electrodynamics, vol. 97 of Springer Proceedings in Physics, edited by: Russer, P. and Mongiardo, M., Springer, Berlin, Heidelberg, 43-54 doi:10.1007/978-3-66207221-9_5, 2004.

Lytle, V. and Jezek, K.: Dielectric permittivity and scattering measurements of Greenland firn at $26.5-40 \mathrm{GHz}$, IEEE T. Geosci. Remote, 32, 290-295, doi:10.1109/36.295044, 1994.

Marti, R., Gascoin, S., Berthier, E., de Pinel, M., Houet, T., and Laffly, D.: Mapping snow depth in open alpine terrain from stereo satellite imagery, The Cryosphere, 10, 1361-1380, doi:10.5194/tc-10-1361-2016, 2016. 
Matrosov, S. Y., Reinking, R. F., and Djalalova, I. V.: Inferring Fall Attitudes of Pristine Dendritic Crystals from Polarimetric Radar Data, J. Atmos. Sci., 1, 241-250, doi:10.1175/JAS-3356.1, 2005.

Matsuoka, K., Wilen, L., Hurley, S., and Raymond, C.: Effects of Birefringence Within Ice Sheets on Obliquely Propagating Radio Waves, IEEE T. Geosci. Remote, 47, 1429-1443, doi:10.1109/TGRS.2008.2005201, 2009.

Matsuoka, T., Fujita, S., and Mae, S.: Effect of temperature on dielectric properties of ice in the range 5-39 GHz, J. Appl. Phys., 80, 5884-5890, doi:10.1063/1.363582, 1996.

Matsuoka, T., Fujita, S., Morishima, S., and Mae, S.: Precise measurement of dielectric anisotropy in ice Ih at $39 \mathrm{GHz}$, J. Appl. Phys., 81, 2344-2348, doi:10.1063/1.364238, 1997.

Mätzler, C.: Applications of the interaction of microwaves with the natural snow cover, Remote Sens. Rev., 2, 259-387, doi:10.1080/02757258709532086, 1987.

Mätzler, C.: Microwave permittivity of dry snow, IEEE T. Geosci. Remote, 34, 573-581, doi:10.1109/36.485133, 1996.

Mätzler, C.: Autocorrelation functions of granular media with free arrangement of spheres, spherical shells or ellipsoids, J. Appl. Phys., 81, 1509-1517, doi:10.1063/1.363916, 1997.

Mätzler, C.: Relation between grain-size and correlation length of snow, J. Glaciol., 48, 461-466, doi:10.3189/172756502781831287, 2002.

Mätzler, C. and Wegmüller, U.: Dielectric properties of freshwater ice at microwave frequencies, J. Phys. D, 20, 1623, doi:10.1088/0022-3727/20/12/013, 1987.

Noel, V. and Chepfer, H.: A global view of horizontally oriented crystals in ice clouds from Cloud-Aerosol Lidar and Infrared Pathfinder Satellite Observation (CALIPSO), J. Geophys. Res.Atmos., 115, 1-13, doi:10.1029/2009JD012365, 2010.

Oh, Y., Sarabandi, K., and Ulaby, F. T.: Semi-empirical model of the ensemble-averaged differential Mueller matrix for microwave backscattering from bare soil surfaces, IEEE T. Geosci. Remote, 40, 1348-1355, doi:10.1109/TGRS.2002.800232, 2002.

Parrella, G., Hajnsek, I., and Papathanassiou, K. P.: Polarimetric Decomposition of L-Band PolSAR Backscattering Over the Austfonna Ice Cap, IEEE T. Geosci. Remote, 54, 1267-1281, doi:10.1109/TGRS.2015.2477168, 2016.

Pfeffer, W. T. and Mrugala, R.: Temperature gradient and initial snow density as controlling factors in the formation and structure of hard depth hoar, J. Glaciol., 48, 485-494, doi:10.3189/172756502781831098, 2002.

Polder, D. and van Santen, J.: The effective permeability of mixtures of solids, Physica, 12, 257-271, doi:10.1016/S00318914(46)80066-1, 1946.

Proksch, M., Löwe, H., and Schneebeli, M.: Density, specific surface area and correlation length of snow measured by highresolution penetrometry, J. Geophys. Res.-Ea. Surf., 120, 346362, doi:10.1002/2014JF003266, 2015.

Rechtsman, M. C. and Torquato, S.: Effective dielectric tensor for electromagnetic wave propagation in random media, J. Appl. Phys., 103, 084901, doi:10.1063/1.2906135, 2008.

Riche, F., Montagnat, M., and Schneebeli, M.: Evolution of crystal orientation in snow during temperature gradient metamorphism, J. Glaciol., 59, 47-55, doi:10.3189/2013JoG12J116, 2013.

Saleh, B. E. A. and Teich, M. C.: Fundamentals of Photonics, John Wiley \& Sons, Inc., New York, 193-237, 1991.
Sarabandi, K.: Derivation of phase statistics from the Mueller matrix, Radio Science, 27, 553-560, doi:10.1029/92RS00195, 1992.

Schneebeli, M. and Sokratov, S.: Tomography of temperature gradient metamorphism of snow and associated changes in heat conductivity, Hydrol. Process., 18, 3655-3665, doi:10.1002/hyp.5800, 2004.

Sihvola, A.: Mixing rules with complex dielectric coefficients, Subsurf. Sens. Technol. Appl., 1, 393-415, doi:10.1023/A:1026511515005, 2000.

Sihvola, A.: How strict are theoretical bounds for dielectric properties of mixtures?, IEEE T. Geosci. Remote, 40, 880-886, doi:10.1109/TGRS.2002.1006369, 2002.

Stangl, M., Werninghaus, R., Schweizer, B., Fischer, C., Brandfass, M., Mittermayer, J., and Breit, H.: TerraSAR-X technologies and first results, IEEE Proc. Radar Sonar Navig., 153, 8695, doi:10.1049/ip-rsn:20045119, 2006.

Sugiyama, S., Enomoto, H., Fujita, S., Fukui, K., Nakazawa, F., and Holmlund, P.: Dielectric permittivity of snow measured along the route traversed in the Japanese-Swedish Antarctic Expedition 2007/08, Ann. Glaciol., 51, 9-15, doi:10.3189/172756410791392745, 2010.

Torquato, S.: Random heterogeneous materials, Springer, New York, 2002.

Torquato, S. and Lado, F.: Trapping constant, thermal conductivity, and the microstructure of suspensions of oriented spheroids, J. Chem. Phys., 94, 4453-4462, doi:10.1063/1.460635, 1991.

Tsang, L.: Polarimetic Passive Microwave Remote Sensing of Random Discrete Scatterers and Rough Surfaces, J. Eelectromag. Waves Appl., 5, 41-57, 1991.

Tsang, L., Pan, J., Liang, D., Li, Z., Cline, D., and Tan, Y.: Modeling Active Microwave Remote Sensing of Snow Using Dense Media Radiative Transfer (DMRT) Theory With MultipleScattering Effects, IEEE T. Geosci. Remote, 45, 990-1004, doi:10.1109/TGRS.2006.888854, 2007.

Tyynelä, J. and Chandrasekar, V.: Characterizing falling snow using multifrequency dual-polarization measurements, J. Geophys Res.-Atmos., 119, 8268-8283, doi:10.1002/2013JD021369, 2014.

Ulaby, F., Held, D., Donson, M., McDonald, K., and Senior, T.: Relating polarization phase difference of SAR signals to scene properties, IEEE T. Geosci. Remote, GE-25, 83-92, doi:10.1109/TGRS.1987.289784, 1987.

Vallese, F. and Kong, J. A.: Correlation function studies for snow and ice, J. Appl. Phys., 52, 4921-4925, doi:10.1063/1.329453, 1981.

Warren, S. G. and Brandt, R. E.: Optical constants of ice from the ultraviolet to the microwave: A revised compilation, J. Geophys. Res.-Atmos., 113, 1-10, doi:10.1029/2007JD009744, 2008.

Weber Hoen, E. and Zebker, H.: Penetration depths inferred from interferometric volume decorrelation observed over the Greenland ice sheet, IEEE T. Geosci. Remote, 38, 2571-2583, doi:10.1109/36.885204, 2000.

Werner, C., Wiesmann, A., Strozzi, T., Schneebeli, M., and Mätzler, C.: The SnowScat ground-based polarimetric scatterometer: Calibration and initial measurements from Davos Switzerland, in: 2010 IEEE International Geoscience and Remote Sensing Symposium (IGARSS), 23-27 July 2010, Honolulu, Hawaii, 23632366, doi:10.1109/IGARSS.2010.5649015, 2010. 
Werninghaus, R. and Buckreuss, S.: The TerraSAR-X Mission and System Design, IEEE T. Geosci. Remote, 48, 606-614, doi:10.1109/TGRS.2009.2031062, 2010.

West, R., Tsang, L., and Winebrenner, D. P.: Dense medium radiative transfer theory for two scattering layers with a Rayleigh distribution of particle sizes, IEEE T. Geosci. Remote, 31, 426-437, 1993.

Wiesmann, A. and Mätzler, C.: Microwave Emission Model of Layered Snowpacks, Remote Sens. Environ., 70, 307-316, doi:10.1016/S0034-4257(99)00046-2, 1999.

Wiesmann, A. and Werner, C.: SnowScat, X- to Ku-Band Scatterometer Development: D13 SnowScat User Manual, Tech. Rep., ESTEC/AO1-5311/06/NL/EL, ESA ESTEC, Gamma Remote Sensing, Gümlingen, Switzerland, 2010.
Wiesmann, A., Werner, C., Mätzler, C., Schneebeli, M., Strozzi, T., and Wegmüller, U.: Mobile $\mathrm{X}$ - to Ku-band Scatterometer in Support of the CoRe-H20 Mission, in: 2008 IEEE International Geoscience and Remote Sensing Symposium, IGARSS 2008, vol. 5, 6-11 July 2008, Boston, USA, V-244V-247, doi:10.1109/IGARSS.2008.4780073, 2008.

Woodcock, N.: Specification of fabric shapes using an eigenvalue method, Geol. Soc. Am. Bull., 88, 1231-1236, 1977.

Xie, X., Löhnert, U., Kneifel, S., and Crewell, S.: Snow particle orientation observed by ground-based microwave radiometry, J. Geophys. Res.-Atmos., 117, 1-12, doi:10.1029/2011JD016369, 2012. 